\title{
Computations of Nambu-Poisson Cohomologies: Case of Nambu-Poisson Tensors of Order 3 on $\mathbb{R}^{4}$
}

By

\author{
Nobutada NAKANISHI*
}

\begin{abstract}
We compute Nambu-Poisson cohomology for Nambu-Poisson tensors of order three which are defined on $\mathbb{R}^{4}$. In particular, we prove that Nambu-Poisson cohomology of exact Nambu-Poisson tensors is equivalent to relative cohomology.
\end{abstract}

\section{$\S 1$. Introduction}

A Nambu-Poisson structure was given by L. Takhtajan [14] in 1994 in order to extend Nambu mechanics defined on $\mathbb{R}^{3}$ to Nambu-Poisson mechanics defined on an $n$-dimensional manifold, $n \geq 3$. One of the main objects of NambuPoisson geometry is to study Nambu-Poisson cohomology and its related topics. The notion of Nambu-Poisson cohomology was first introduced by R. Ibáñez et al. [7], and it is an extension of Poisson cohomology (or Lichnerowicz-Poisson cohomology) on a Poisson manifold. Let $(M, \eta)$ be an $m$-dimensional NambuPoisson manifold. (See Definition 2.1 for the precise definition.) Whenever we mention a Nambu-Poisson manifold, $m$ is assumed to be $m \geq 3$. Then a Nambu-Poisson tensor $\eta$ defines the so-called characteristic foliation, which is, in general, a singular foliation on $M$. In case that $\eta$ is a Nambu-Poisson tensor, then the set of Hamiltonian vector fields becomes a Lie subalgebra of $\chi(M)$,

Communicated by K. Saito. Received February 2004. Revised July 29, 2004, December 9, 2004.

2000 Mathematics Subject Classification(s): 53D, 17.

Key words: Nambu-Poisson tensor, Nambu-Poisson cohomology, relative cohomology.

*Department of Mathematics, Gifu Keizai University, 5-50 Kitagata, Ogaki-city, Gifu, 503-8550, Japan.

e-mail: nakanisi@gifu-keizai.ac.jp

(C) 2006 Research Institute for Mathematical Sciences, Kyoto University. All rights reserved. 
the Lie algebra of all vector fields on $M$. This Lie subalgebra will be denoted by $\mathcal{H}$.

Let $\Omega^{k}(M)$ be the space of $k$-forms on $M$, and let the order of $\eta$ be $n$. (i.e. $\eta \in \Gamma\left(\Lambda^{n} T M\right)$, where $\Gamma\left(\Lambda^{n} T M\right)$ is the space of cross-sections $M \longrightarrow \Lambda^{n} T M$.) Here $m \geq n \geq 3$, and $n \geq k$. We define a mapping

$$
\sharp_{k}: \Omega^{k}(M) \longrightarrow \Gamma\left(\Lambda^{n-k} T M\right)
$$

by $\sharp_{k}(\alpha)=i(\alpha) \eta$ for $\alpha \in \Omega^{k}(M)$. If $k=n-1, \Omega^{n-1}(M)$ has a structure of Leibniz algebra, which is defined by

$$
\{\alpha, \beta\}=\mathcal{L}_{\sharp_{n-1}(\alpha)} \beta+(-1)^{n} \sharp_{n}(d \alpha) \beta, \quad \alpha, \beta \in \Omega^{n-1}(M),
$$

where $\mathcal{L}$ stands for the Lie derivative. The image of $\sharp_{n-1}$, which is denoted by $\mathfrak{g}$, becomes a Lie subalgebra of $\chi(M)$. (See Proposition 3.1 and its explanation.) It is clear that $\mathcal{H}$ is contained in $\mathfrak{g}$. Nambu-Poisson cohomology is a cohomology group of a Lie algebra $\mathfrak{g}$ having $C^{\infty}(M, \mathbb{R})$ as its representation space, which is also called Chevalley-Eilenberg cohomology of $\mathfrak{g}$. It will be denoted by $H_{N P}^{*}$. It is easy to see that $H_{N P}^{0}$ is equal to the space of invariant functions of $\mathfrak{g}$. Moreover $H_{N P}^{1}$ is deeply related to the modular class of $(M, \eta)$ [7]. It will be expected that other cohomologies $H_{N P}^{*}$ have also some geometric meanings.

If $\eta$ does not vanish anywhere on $M$, it is said to be regular. Then R. Ibáñez et al. computed Nambu-Poisson cohomology of a regular Nambu-Poisson manifold $(M, \eta)$ [7]. If $\eta$ has some singularities, it is quite difficult to compute its Nambu-Poisson cohomology. As an example of a singular Nambu-Poisson manifold, they also considered $\left(\mathbb{R}^{3}, \eta=\left(x^{2}+y^{2}+z^{2}\right) \frac{\partial}{\partial x} \wedge \frac{\partial}{\partial y} \wedge \frac{\partial}{\partial z}\right)$, and they proved that the first Nambu-Poisson cohomology group $H_{N P}^{1}\left(\mathbb{R}^{3}, \eta\right)$ is isomorphic to $\mathbb{R}$.

On the other hand, P. Monnier [9] computed Nambu-Poisson cohomology for germs at 0 of $n$-vectors $\eta=f \frac{\partial}{\partial x_{1}} \wedge \cdots \wedge \frac{\partial}{\partial x_{n}}$ on $\mathbb{K}^{n}(\mathbb{K}=\mathbb{R}$ or $\mathbb{C})$, with the assumption that $f$ is a quasihomogeneous polynomial of finite codimension. His results contain the result of $\mathrm{R}$. Ibáñez et al., (at least in the formal case).

As the next step, it is natural to consider the case that the order of a Nambu-Poisson tensor $\eta$ is smaller than the dimension of a space on which $\eta$ is defined. In the present paper, along this concept, we will compute NambuPoisson cohomology for the following three cases.

(a) Exact Nambu-Poisson tensors $\eta$ of order 3 defined on $\mathbb{R}^{4}(x, y, z, u)$. A Nambu-Poisson tensor $\eta$ is called exact if there is a function $f$ such that $i(\eta) \Omega=d f$ for $\Omega=d x \wedge d y \wedge d z \wedge d u$.

(b) Linear Nambu-Poisson tensors of order 3 defined on $\mathbb{R}^{4}(x, y, z, u)$. 
(c) A quadratic Nambu-Poisson tensor $\eta=\left(x^{2}+y^{2}+z^{2}+u^{2}\right) \frac{\partial}{\partial x} \wedge \frac{\partial}{\partial y} \wedge \frac{\partial}{\partial z}$ of order 3 defined on $\mathbb{R}^{4}(x, y, z, u)$.

The computation for the case (a) naturally leads us to the notion of relative cohomology which was studied by C. A. Roche [13]. In this case, we know that $H_{N P}^{k}=H_{r e l}^{k}$ for $0 \leq k \leq 2$. In computing Nambu-Poisson cohomology of the case (b), we will use the classification theorem of linear Nambu-Poisson tensors which was proved by J-P. Dufour and N. T. Zung [3]. A part of this case is also discussed in (a). In treating the case (c), we will take advantage of the results of P. Monnier [9].

Here we computed Nambu-Poisson cohomology only for the case $\left(\mathbb{R}^{4}, \eta\right)$, where the order of $\eta$ is three. But it is not so difficult to extend all the results we have obtained here to more general situations. In fact let us consider a Nambu-Poisson manifold $\left(\mathbb{R}^{n}, \eta\right)$, where the order of $\eta$ is $n^{\prime}$. We can easily see that if $n-n^{\prime}>1$, then spaces of cohomologies are, in general, greater than those of the case $n-n^{\prime}=1$. This is because that the space of $\mathfrak{g}$-invariant functions becomes greater if $n-n^{\prime}>1$.

The author would like to express his deep thanks to Professors T.Fukuda, H. Sato, Y. Agaoka and G. Ishikawa for helpful and stimulating discussions. $\mathrm{He}$ is also grateful to $\mathrm{P}$. Monnier for valuable communications.

\section{§2. Reviews of Nambu-Poisson Manifolds}

We will review some useful results of geometry of Nambu-Poisson manifolds. Details are referred to [7],[10] and [14]. Let $M$ be an $m$-dimensional $C^{\infty}$-manifold, and $\mathcal{F}$ its algebra of real valued $C^{\infty}$-functions on $M$. We denote by $\Gamma\left(\Lambda^{n} T M\right)$ the space of global cross-sections $\eta: M \longrightarrow \Lambda^{n} T M$. Then for each $\eta \in \Gamma\left(\Lambda^{n} T M\right)$, there corresponds the bracket defined by

$$
\left\{f_{1}, \ldots, f_{n}\right\}=\eta\left(d f_{1}, \ldots, d f_{n}\right), \quad f_{1}, \ldots, f_{n} \in \mathcal{F} .
$$

This bracket operation is an $n$-linear skew-symmetric map from $\mathcal{F}^{n}$ to $\mathcal{F}$ which satisfies the Leibniz rule:

$$
\left\{f_{1}, \ldots, f_{n-1}, g_{1} \cdot g_{2}\right\}=\left\{f_{1}, \ldots, f_{n-1}, g_{1}\right\} \cdot g_{2}+g_{1} \cdot\left\{f_{1}, \ldots, f_{n-1}, g_{2}\right\},
$$

for all $f_{1}, \ldots, f_{n-1}, g_{1}, g_{2} \in \mathcal{F}$.

Let $A=\sum f_{i_{1}} \wedge \cdots \wedge f_{i_{n-1}}, f_{i_{j}} \in \mathcal{F}$. Since the bracket operation satisfies the Leibniz rule, we can define a vector field $X_{A}$ corresponding to $A$ by the following equation:

$$
X_{A}(g)=\sum\left\{f_{i_{1}}, \ldots, f_{i_{n-1}}, g\right\}, \quad g \in \mathcal{F} .
$$


Such a vector field is called a Hamiltonian vector field. The space of Hamiltonian vector fields is denoted by $\mathcal{H}$.

Definition 2.1. $\quad \eta \in \Gamma\left(\Lambda^{n} T M\right)$ is called a Nambu-Poisson tensor of order $n$ if it satisfies $\mathcal{L}_{X_{A}} \eta=0$ for all $X_{A} \in \mathcal{H}$, where $\mathcal{L}$ is the Lie derivative. Then a Nambu-Poisson manifold is a pair $(M, \eta)$.

Let $\eta(p) \neq 0, p \in M$. Then we say that $\eta$ is regular at $p$. Now we can state the following local structure theorem for Nambu-Poisson tensors [5],[10].

Theorem 2.1. $\quad$ Let $\eta \in \Gamma\left(\Lambda^{n} T M\right), n \geq 3$. If $\eta$ is a Nambu-Poisson tensor of order $n$, then for any regular point $p$, there exists a coordinate neighborhood $U$ with local coordinates $\left(x_{1}, \ldots, x_{n}, x_{n+1}, \ldots, x_{m}\right)$ around $p$ such that

$$
\eta=\frac{\partial}{\partial x_{1}} \wedge \cdots \wedge \frac{\partial}{\partial x_{n}}
$$

on $U$, and vice versa.

Let $(M, \eta)$ be a Nambu-Poisson manifold with volume form $\Omega$, and $m \geq$ $n \geq 3$. Put $\omega=i(\eta) \Omega$, where the right hand side is the interior product of $\eta$ and $\Omega$. Hence $\omega$ is an $(m-n)$-form. The following theorem gives a necessary and sufficient condition for $\eta$ to be a Nambu-Poisson tensor. For the proof, see [11].

Theorem 2.2. Let $\eta \in \Gamma\left(\Lambda^{n} T M\right)$. Then $\eta$ is a Nambu-Poisson tensor if and only if $\eta$ satisfies the following two conditions around each regular point:

(a) $\omega$ is (locally) decomposable, and

(b) there exists a locally defined 1-form $\theta$ such that $d \omega=\theta \wedge \omega$.

\section{§3. Nambu-Poisson Cohomology}

Let $(M, \eta)$ be a Nambu-Poisson manifold of order $n$ and let $k$ be an integer with $k \leq n$. Denote by $\Omega^{k}(M)$ the space of $k$-forms on $M$. If $\Lambda^{k}\left(T^{*} M\right)$ (respectively, $\Lambda^{n-k}(T M)$ ) denotes the vector bundle of the $k$-forms (respectively, $(n-k)$-vectors) then $\eta$ induces a homomorphism of vector bundles $\sharp_{k}: \Lambda^{k}\left(T^{*} M\right) \rightarrow \Lambda^{n-k}(T M)$ by defining

$$
\sharp_{k}(\beta)=i(\beta) \eta(x)
$$


for $\beta \in \Lambda^{k}\left(T_{x}^{*} M\right)$ and $x \in M$, where $i(\beta)$ is the contraction by $\beta$. Denote also by $\sharp_{k}$ the homomorphism of $\mathcal{F}$-modules from the space $\Omega^{k}(M)$ into the space $\Gamma\left(\Lambda^{n-k} T M\right)$ given by

$$
\sharp_{k}(\alpha)(x)=\sharp_{k}(\alpha(x))
$$

for all $\alpha \in \Omega^{k}(M)$ and $x \in M$.

Next we define a Leibniz algebra structure on $\Omega^{n-1}(M)$. The Leibniz algebra on $\Omega^{n-1}(M)$ attached to $M$ is the bracket of $(n-1)$-forms $\{$,$\} :$ $\Omega^{n-1}(M) \times \Omega^{n-1}(M) \rightarrow \Omega^{n-1}(M)$ defined by

$$
\{\alpha, \beta\}=\mathcal{L}_{\sharp_{n-1}(\alpha)} \beta+(-1)^{n} \sharp_{n}(d \alpha) \beta
$$

for all $\alpha, \beta \in \Omega^{n-1}(M)$. In particular, we have that

$$
\sharp_{n-1}(\{\alpha, \beta\})=\left[\sharp_{n-1}(\alpha), \sharp_{n-1}(\beta)\right]
$$

for all $\alpha, \beta \in \Omega^{n-1}(M)$.

Using Theorem 2.1, the following proposition was proved by R. Ibáñez et al. [7].

Proposition 3.1. Let $(M, \eta)$ be an m-dimensional Nambu-Poisson manifold of order $n$, with $n \geq 3$. Then the center of the algebra $\left(\Omega^{n-1}(M),\{\},\right)$ is the $\mathcal{F}$-module

$$
\operatorname{ker} \sharp_{n-1}=\left\{\alpha \in \Omega^{n-1}(M) \mid \sharp_{n-1}(\alpha)=0\right\} .
$$

By the above proposition, we know that $\Omega^{n-1}(M) /$ ker $\sharp_{n-1}$ is isomorphic to a Lie subalgebra of $\chi(M)$. This Lie algebra is often denoted by $\mathfrak{g}$. And $\mathcal{F}$ is a $\left(\Omega^{n-1}(M) /\right.$ ker $\left.\sharp_{n-1}\right)$-module relative to the representation:

$$
\Omega^{n-1}(M) / \operatorname{ker} \sharp_{n-1} \times \mathcal{F} \longrightarrow \mathcal{F}, \quad([\alpha], f) \mapsto[\alpha] f=\left(\sharp_{n-1}(\alpha)\right)(f) .
$$

According to [7], one can define the skew symmetric-cochain complex

$$
\left(C^{*}\left(\Omega^{n-1}(M) / \operatorname{ker} \sharp_{n-1} ; \mathcal{F}\right)=\bigoplus_{k} C^{k}\left(\Omega^{n-1}(M) / \operatorname{ker} \sharp_{n-1} ; \mathcal{F}\right), \partial\right)
$$

where the space of the $k$-cochains $C^{k}\left(\Omega^{n-1}(M) /\right.$ ker $\left.\sharp_{n-1} ; \mathcal{F}\right)$ consists of skewsymmetric $\mathcal{F}$-linear mappings

$$
c^{k}:\left(\Omega^{n-1}(M) / \operatorname{ker} \sharp_{n-1}\right) \times \cdots \times\left(\Omega^{n-1}(M) / \operatorname{ker} \sharp_{n-1}\right) \rightarrow \mathcal{F}
$$


and the coboundary operator $\partial$ is given by

$$
\begin{aligned}
\partial c^{k}( & {\left.\left[\alpha_{0}\right], \ldots,\left[\alpha_{k}\right]\right)=\sum_{i=0}^{k}(-1)^{i}\left(\sharp_{n-1}\left(\alpha_{i}\right)\right)\left(c^{k}\left(\left[\alpha_{0}\right], \ldots, \widehat{\left[\alpha_{i}\right]}, \ldots,\left[\alpha_{k}\right]\right)\right) } \\
& +\sum_{0 \leq i<j \leq k}(-1)^{i+j} c^{k}\left(\left[\left\{\alpha_{i}, \alpha_{j}\right\}\right],\left[\alpha_{0}\right], \ldots, \widehat{\left[\alpha_{i}\right]}, \ldots, \widehat{\left[\alpha_{j}\right]}, \ldots,\left[\alpha_{k}\right]\right)
\end{aligned}
$$

for all $c^{k} \in C^{k}\left(\Omega^{n-1}(M) / \operatorname{ker} \sharp_{n-1} ; \mathcal{F}\right)$, and $\left[\alpha_{0}\right], \ldots,\left[\alpha_{k}\right] \in \Omega^{n-1}(M) / \operatorname{ker} \sharp_{n-1}$. Then we have $\partial \circ \partial=0$. The cohomology of this complex is called NambuPoisson cohomology and denoted by $H_{N P}^{*}(M, \eta)$.

Remark 3.1. Since a Nambu-Poisson tensor $\eta$ satisfies $[\eta, \eta]=0$ (Schouten bracket), we can define three cohomology spaces $H_{\eta}^{0}(M), H_{\eta}^{1}(M)$ and $H_{\eta}^{2}(M)$ as in the case of usual Poisson manifolds. We see that these three spaces appear as parts of Nambu-Poisson cohomology spaces. (See [9].)

The first attempt at the computation of singular Nambu-Poisson cohomology was carried out by R. Ibáñez et al. In [7], they considered a NambuPoisson manifold $\left\{\mathbb{R}^{3}, \eta=\left(x^{2}+y^{2}+z^{2}\right) \frac{\partial}{\partial x} \wedge \frac{\partial}{\partial y} \wedge \frac{\partial}{\partial z}\right\}$. They obtained that $H_{N P}^{1}\left(\mathbb{R}^{3}, \eta\right) \cong \mathbb{R}$.

In [9], P. Monnier studied Nambu-Poisson cohomology from slightly more general viewpoint, which includes the case of R. Ibáñez et al. [7]. That is to say, he computed Nambu-Poisson cohomology of Nambu-Poisson manifolds of the form $\left(\mathbb{R}^{n}, \eta=f \frac{\partial}{\partial x_{1}} \wedge \cdots \wedge \frac{\partial}{\partial x_{n}}\right)$, where $f$ is a quasihomogeneous polynomial of finite codimension. Using his results, we compute Nambu-Poisson cohomology of $\left(\mathbb{R}^{4}, \eta=\left(x^{2}+y^{2}+z^{2}+u^{2}\right) \frac{\partial}{\partial x} \wedge \frac{\partial}{\partial y} \wedge \frac{\partial}{\partial z}\right)$ in the last section.

\section{§4. Computation of Nambu-Poisson Cohomology: Exact Case}

\section{$\S 4.1 . \quad$ Notation and general remarks}

Let $\mathcal{F}$ be the space of $C^{\infty}$-functions on $\mathbb{R}^{4}$. Throughout this section, we suppose that $\mathcal{F} \ni f$ satisfies $f(0)=0$, and is of finite codimension, which means that $\mathcal{F} /\langle f\rangle\left(\langle f\rangle\right.$ is the ideal spanned by $\left.f_{x}, f_{y}, f_{z}, f_{u}\right)$ is a finite dimensional vector space. Here we simply write, for example, $f_{x}$ for $\frac{\partial f}{\partial x}$.

Let $\eta$ be a Nambu-Poisson tensor of order 3 on $\mathbb{R}^{4}(x, y, z, u)$. $\eta$ is said to be exact if $\eta$ satisfies $i(\eta) \Omega=d f$, where $\Omega=d x \wedge d y \wedge d z \wedge d u$. Then $\eta$ is written as follows.

$\eta=-f_{x} \frac{\partial}{\partial y} \wedge \frac{\partial}{\partial z} \wedge \frac{\partial}{\partial u}+f_{y} \frac{\partial}{\partial x} \wedge \frac{\partial}{\partial z} \wedge \frac{\partial}{\partial u}-f_{z} \frac{\partial}{\partial x} \wedge \frac{\partial}{\partial y} \wedge \frac{\partial}{\partial u}+f_{u} \frac{\partial}{\partial x} \wedge \frac{\partial}{\partial y} \wedge \frac{\partial}{\partial z}$. 
A Lie subalgebra $\mathfrak{g}=\sharp_{2}\left(\Omega^{2}\left(\mathbb{R}^{4}\right)\right)$ of $\chi\left(\mathbb{R}^{4}\right)$ is spanned over $\mathcal{F}$ by the following six vector fields.

$$
\begin{cases}X_{1}=f_{x} \frac{\partial}{\partial y}-f_{y} \frac{\partial}{\partial x}, & X_{2}=f_{x} \frac{\partial}{\partial z}-f_{z} \frac{\partial}{\partial x}, \quad X_{3}=f_{x} \frac{\partial}{\partial u}-f_{u} \frac{\partial}{\partial x} \\ X_{4}=f_{y} \frac{\partial}{\partial z}-f_{z} \frac{\partial}{\partial y}, & X_{5}=f_{y} \frac{\partial}{\partial u}-f_{u} \frac{\partial}{\partial y}, \quad X_{6}=f_{z} \frac{\partial}{\partial u}-f_{u} \frac{\partial}{\partial z}\end{cases}
$$

It is easy to see that $\Lambda^{4} \mathfrak{g}=0$. Hence $H_{N P}^{k}=0$, for $k \geq 4$.

\section{§4.2. Relative cohomology}

In this subsection, we show that Nambu-Poisson cohomology of exact Nambu-Poisson structure is equivalent to relative cohomology which was studied by C. A. Roche [13].

In the first half of this subsection, all objects are considered on $\mathbb{R}^{s}$. And we simply write $\Omega^{k}$ for $\Omega^{k}\left(\mathbb{R}^{s}\right)$. Suppose that $C^{\infty}\left(\mathbb{R}^{s}\right) \ni f$ satisfies $f(0)=0$ and is of finite codimension. That is to say, an ideal generated by coefficients of $d f$ is of finite codimension in $C^{\infty}\left(\mathbb{R}^{s}\right)$.

First note that $d f \wedge \Omega^{k}$ is compatible with the exterior differential $d$ : i.e., $d\left(d f \wedge \Omega^{k-1}\right) \subset d f \wedge \Omega^{k}$. Hence the linear mapping

$$
d_{r e l}: \Omega^{k} / d f \wedge \Omega^{k-1} \longrightarrow \Omega^{k+1} / d f \wedge \Omega^{k}
$$

is well-defined.

Definition 4.1. The following sequence defined on $\mathbb{R}^{s}$ is called relative complex of $f$.

$$
0 \longrightarrow \Omega^{0} \stackrel{d_{r e l}}{\longrightarrow} \Omega^{1} / d f \wedge \Omega^{0} \stackrel{d_{r e l}}{\longrightarrow} \Omega^{2} / d f \wedge \Omega^{1} \stackrel{d_{r e l}}{\longrightarrow} \cdots \stackrel{d_{r e l}}{\longrightarrow} \Omega^{s} / d f \wedge \Omega^{s-1} \longrightarrow 0
$$

The cohomology of complex defined above is called relative cohomlogy of $f$, and is denoted by $H_{r e l}^{*}(f)$ or $H_{r e l}^{*}$. In the above sequence, if we put $\mathcal{I} \cdot \Omega^{k}$ into $\Omega^{k}$, then we have flat relative cohomology $H_{\infty \text { rel }}^{k}$, where $\mathcal{I}$ denotes the space of flat functions of $\mathcal{F}$ at the origin. Moreover if we consider formal differential $k$-forms instead of $\Omega^{k}$, we have formal relative cohomology $\hat{H}_{r e l}^{k}$.

To state the structure of $H_{\infty \text { rel }}^{k}$ it is convenient to introduce the following notations: For a positive small number $c$,

$$
\begin{aligned}
& b_{+}^{k}=\operatorname{dim} H^{k}\left(X_{+c}, \mathbb{R}\right), \quad b_{-}^{k}=\operatorname{dim} H^{k}\left(X_{-c}, \mathbb{R}\right), \\
& m^{\infty}(1)=\text { the space of flat functions at the origin of 1-variable, } \\
& m_{ \pm}^{\infty}=\left\{h \in m^{\infty}(1) \mid h\left(\mathbb{R}^{\mp}\right)=0\right\}, \\
& X_{ \pm c}=f^{-1}( \pm c) \cap B, \text { where } B \text { is a small ball centered at the origin. }
\end{aligned}
$$


Then C. A. Roche [13] proved the following theorems. All objects are considered on $\mathbb{R}^{s}$.

Theorem 4.1. The $m^{\infty}(1)$-module $H_{\infty \text { rel }}^{k}$ is isomorphic to $\left(m_{+}^{\infty}\right)^{b_{+}^{k}} \times$ $\left(m_{-}^{\infty}\right)^{b_{-}^{k}}$.

Theorem 4.2. $\quad$ There are the following mutual relations among three cohomologies.

$$
\begin{aligned}
& H_{\text {rel }}^{k} \cong H_{\infty \text { rel }}^{k} \text { if } 0<k<s-1 \\
& H_{\text {rel }}^{0} / H_{\infty \text { rel }}^{0} \cong \mathcal{F}(1), H_{\text {rel }}^{s-1} / H_{\infty \text { rel }}^{s-1} \cong \hat{H}_{\text {rel }}^{s-1} \cong \mathcal{F}(1)^{\mu},
\end{aligned}
$$

where $\mathcal{F}(1)$ is the space of formal functions of 1-variable, and $\mu=$ codim $f$. $\mathcal{F}(1)^{\mu}$ denotes the free $\mathcal{F}(1)$-module of rank $\mu$.

In the latter half of this subsection, let us return to the case of $\mathbb{R}^{4}$. We simply write $\Omega^{k}$ for $\Omega^{k}\left(\mathbb{R}^{4}\right)$.

Definition 4.2. We define the subspace $I^{k}$ of $\Omega^{k}$ by

$$
I^{k}=\left\{c \in \Omega^{k} \mid c(\overbrace{\mathfrak{g}, \ldots, \mathfrak{g}}^{k})=0\right\},
$$

for $1 \leq k \leq 4$. Put $I^{0}=0$.

It is clear that $I^{4}=\Omega^{4}$ since $\Lambda^{4} \mathfrak{g}=0$. In the rest of this subsection, we give a characterization of $I^{k}$ for $k=1,2,3$.

Proposition 4.3. $\quad I^{k}=\left\{c \in \Omega^{k} \mid c \wedge d f=0\right\}$, for $0 \leq k \leq 4$.

Proof. In case of $k=1$, put $c=A d x+B d y+C d z+D d u \in \Omega^{1}$. Then $c(\mathfrak{g})=0$ implies that $f_{x} B=f_{y} A, f_{x} C=f_{z} A, f_{x} D=f_{u} A, f_{y} C=$ $f_{z} B, f_{z} D=f_{u} C$, and $f_{y} D=f_{u} B$. On the other hand,

$$
\begin{aligned}
c \wedge d f & =(A d x+B d y+C d z+D d u) \wedge\left(f_{x} d x+f_{y} d y+f_{z} d z+f_{u} d u\right) \\
& =\left(f_{y} A-f_{x} B\right) d x \wedge d y+\left(f_{z} A-f_{x} C\right) d x \wedge d z+\left(f_{u} A-f_{x} D\right) d x \wedge d u \\
& +\left(f_{z} B-f_{y} C\right) d y \wedge d z+\left(f_{u} B-f_{y} D\right) d y \wedge d u+\left(f_{u} C-f_{z} D\right) d z \wedge d u .
\end{aligned}
$$

Thus we have that $c(\mathfrak{g})=0$ if and only if $c \wedge d f=0$.

For cases of $k \geq 2$, we can prove in the same way as the case of $k=1$.

Now let us recall G. de Rham's division lemma [2]. We will explain this lemma in the general situation, $s$-dimensional Euclidean space $\mathbb{R}^{s}$. (Our case is, of course, $s=4$.) 
Definition 4.3. An element $\omega$ of $\Omega^{1}$ is said to possess the property of division in $\Omega^{*}$ if for any $\alpha \in \Omega^{p}, 1 \leq p \leq s-1$, which satisfies $\omega \wedge \alpha=0$, there exists $\beta \in \Omega^{p-1}$ such that $\alpha=\omega \wedge \beta$.

Definition 4.4. Let $\omega \in \Omega^{1}$ and let $I(\omega)$ be the ideal of $\Omega^{0}=C^{\infty}\left(\mathbb{R}^{s}\right)$ spanned by the coefficients of $\omega$. Then 0 is said to be algebraically isolated zero of $\omega$ if $\Omega^{0} / I(\omega)$ is a finite dimensional vector space over $\mathbb{R}$.

Lemma 4.4. Let $\omega$ be an element of $\Omega^{1}$. If 0 is algebraically isolated zero of $\omega$, then $\omega$ possesses the property of division.

Since $f$ is of finite codimension in our situation, $\omega=d f$ satisfies the condition of Lemma 4.4. Hence by Proposition 4.3, we know that $I^{k}=d f \wedge \Omega^{k-1}$ for $1 \leq k \leq 3$.

Recall that a $k$-th cochain $c \in C^{k}$ is $\mathcal{F}$-linear skew-symmetric mapping from $\mathfrak{g} \times \cdots \times \mathfrak{g}$ to $\mathcal{F}$. The natural inclusion $\iota: \mathfrak{g} \hookrightarrow \chi\left(\mathbb{R}^{4}\right)$ induces the surjective mapping $\phi: \Omega^{k} \longrightarrow C^{k}$ as the dual mapping of the natural inclusion $\iota$. Note that $\operatorname{ker} \phi=I^{k}$ for $1 \leq k \leq 3$. Then it is easy to obtain the following proposition.

Proposition 4.5. $\quad C^{k} \cong \Omega^{k} / I^{k} \cong \Omega^{k} / d f \wedge \Omega^{k-1}$, for $1 \leq k \leq 3$. For $k=0, C^{0}=\Omega^{0}=\mathcal{F}$, and for $k=4, C^{4}=0$.

Now by Proposition 4.5, we have obtained the following commutative diagram. In particular, note that $d_{r e l}: \Omega^{k} / I^{k} \rightarrow \Omega^{k+1} / I^{k+1}$ coincides with $\partial: C^{k} \rightarrow C^{k+1}$ for $0 \leq k \leq 2$.

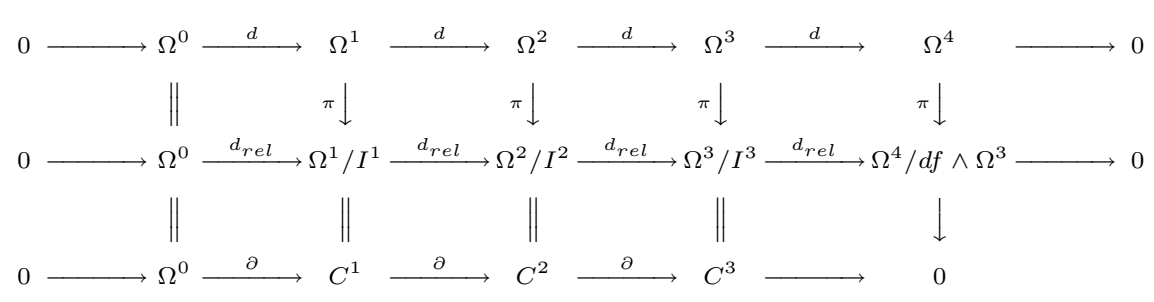

Using the above commutative diagram, we can get the following theorem. 
Theorem 4.6. $\quad$ Let $\eta$ be the exact Nambu-Poisson tensor corresponding to $f \in \mathcal{F}$ defined on $\mathbb{R}^{4}$, where $f$ is of finite codimension. Then

$$
\begin{aligned}
& H_{N P}^{k} \cong H_{\text {rel }}^{k} \text { for } 0 \leq k \leq 2, \\
& H_{N P}^{3} \cong H_{\text {rel }}^{3} \oplus \Omega^{4} / d f \wedge \Omega^{3}, \\
& H_{N P}^{k}=0 \text { for } 4 \leq k .
\end{aligned}
$$

To compute some examples of exact Nambu-Poisson cohomology, let us recall the results of C. A. Roche [13]. (See Theorem 4.1 and Theorem 4.2.)

Examples. Let $f=x^{k}+y^{2}+z^{2}+u^{2}, k \geq 3$. Then if $k$ is an odd positive integer, both $X_{+c}$ and $X_{-c}$ are homeomorphic to $D^{3}$, where $D^{3}$ denotes a three dimensional ball. Hence by Theorem 4.1 and Theorem 4.2, we have

$$
\begin{aligned}
& H_{\infty r e l}^{0} \cong m_{+}^{\infty} \times m_{-}^{\infty}, H_{\infty r e l}^{1}=0, H_{\infty r e l}^{2}=0, \quad H_{\infty r e l}^{3}=0 . \\
& H_{r e l}^{0} \cong C^{\infty}\left(\mathbb{R}^{+}\right) \times C^{\infty}\left(\mathbb{R}^{-}\right), \quad H_{\text {rel }}^{1}=0, \quad H_{r e l}^{2}=0, \quad H_{r e l}^{3} \cong \mathcal{F}(1)^{k-1} .
\end{aligned}
$$

Moreover if we use Theorem 4.6, we have

$H_{N P}^{0} \cong C^{\infty}\left(\mathbb{R}^{+}\right) \times C^{\infty}\left(\mathbb{R}^{-}\right), H_{N P}^{1}=0, \quad H_{N P}^{2}=0, \quad H_{N P}^{3} \cong \mathcal{F}(1)^{k-1} \oplus \mathbb{R}^{k-1}$.

On the other hand, if $k$ is an even positive integer, then $X_{+c}$ is homeomorphic to $S^{3}$ and $X_{-c}=\phi$. Hence we have

$$
\begin{aligned}
& H_{\infty r e l}^{0} \cong m_{+}^{\infty}, H_{\infty r e l}^{1}=0, H_{\infty r e l}^{2}=0, \quad H_{\infty r e l}^{3} \cong m_{+}^{\infty} . \\
& H_{r e l}^{0} \cong C^{\infty}\left(\mathbb{R}^{+}\right), \quad H_{r e l}^{1}=0, \quad H_{r e l}^{2}=0, \quad H_{r e l}^{3} \cong\left(C^{\infty}\left(\mathbb{R}^{+}\right)\right)^{k-1} .
\end{aligned}
$$

Moreover if we use Theorem 4.6, we have

$$
H_{N P}^{0} \cong C^{\infty}\left(\mathbb{R}^{+}\right), H_{N P}^{1}=0, H_{N P}^{2}=0, \quad H_{N P}^{3} \cong\left(C^{\infty}\left(\mathbb{R}^{+}\right)\right)^{k-1} \oplus \mathbb{R}^{k-1} .
$$

\section{§5. Computation of Nambu-Poisson Cohomology: Linear Case}

\section{§5.1. Notation and general remarks}

In this section we consider linear Nambu-Poisson tensors which are of order 3 on $\mathbb{R}^{4}(x, y, z, u)$. By the classification theorem of linear Nambu-Poisson structures [3],[6], we know that there are the following four types of linear Nambu-Poisson tensors.

(I) $\eta=-f_{x} \frac{\partial}{\partial y} \wedge \frac{\partial}{\partial z} \wedge \frac{\partial}{\partial u}+f_{y} \frac{\partial}{\partial x} \wedge \frac{\partial}{\partial z} \wedge \frac{\partial}{\partial u}-f_{z} \frac{\partial}{\partial x} \wedge \frac{\partial}{\partial y} \wedge \frac{\partial}{\partial u}+f_{u} \frac{\partial}{\partial x} \wedge \frac{\partial}{\partial y} \wedge \frac{\partial}{\partial z}$, where $f$ is a homogeneous quadratic function on $\mathbb{R}^{4}$. 
(II) $\eta=\frac{\partial}{\partial x} \wedge \frac{\partial}{\partial y} \wedge\left\{\left(a_{11} z+a_{12} u\right) \frac{\partial}{\partial z}+\left(a_{21} z+a_{22} u\right) \frac{\partial}{\partial u}\right\}, \quad\left(a_{i j} \in \mathbb{R}\right)$.

(III) $\eta_{\phi}=\phi \frac{\partial}{\partial x} \wedge \frac{\partial}{\partial y} \wedge \frac{\partial}{\partial z}$, where $\phi$ is any linear function on $\mathbb{R}^{4}$.

(IV) $\eta_{\psi}=\left\{p x+(q-1) y-b_{3} z-b_{4} u\right\} \frac{\partial}{\partial y} \wedge \frac{\partial}{\partial z} \wedge \frac{\partial}{\partial u}-\left\{(q+1) x+r y+a_{3} z+\right.$ $\left.a_{4} u\right\} \frac{\partial}{\partial x} \wedge \frac{\partial}{\partial z} \wedge \frac{\partial}{\partial u}$, where $p, q, r, a_{3}, a_{4}, b_{3}, b_{4} \in \mathbb{R}$. Put $\alpha=d \psi+\left(x+a_{3} z+\right.$ $\left.a_{4} u\right) d y-\left(y+b_{3} z+b_{4} u\right) d x$, where $\psi=\frac{1}{2} p x^{2}+q x y+\frac{1}{2} r y^{2}$. Then $\eta_{\psi}$ is defined by $i\left(\eta_{\psi}\right) d x \wedge d y \wedge d z \wedge d u=\alpha$.

In (IV), recall that $\eta_{\psi}$ becomes a Nambu-Poisson tensor if and only if $\alpha \wedge d \alpha=0$. Thus seven constants must satisfy $a_{3} b_{4}=a_{4} b_{3}, a_{3} p+b_{3}(q+1)$ $=0, a_{3}(q-1)+b_{3} r=0, a_{4} p+b_{4}(q+1)=0, a_{4}(q-1)+b_{4} r=0$.

In considering type (II), since a matrix $\left(a_{i j}\right)$ can be chosen to be in Jordan form, there are five classes with nondegenerate Jordan forms $\left(\eta_{1} \sim \eta_{5}\right)$ and two classes with degenerate Jordan forms $\left(\eta_{6} \sim \eta_{7}\right)$ as follows.

(i) $\eta_{1}=\frac{\partial}{\partial x} \wedge \frac{\partial}{\partial y} \wedge\left\{(\alpha z+u) \frac{\partial}{\partial z}+(\alpha u) \frac{\partial}{\partial u}\right\}, \quad \alpha \neq 0$,

(ii) $\eta_{2}=\frac{\partial}{\partial x} \wedge \frac{\partial}{\partial y} \wedge\left\{(\alpha z) \frac{\partial}{\partial z}+(\beta u) \frac{\partial}{\partial u}\right\}, \quad \alpha \neq 0, \beta \neq 0, \alpha \neq \beta$,

(iii) $\eta_{3}=\frac{\partial}{\partial x} \wedge \frac{\partial}{\partial y} \wedge\left\{(\alpha z-\beta u) \frac{\partial}{\partial z}+(\beta z+\alpha u) \frac{\partial}{\partial u}\right\}, \quad \alpha \neq 0 \beta \neq 0$,

(iv) $\eta_{4}=\frac{\partial}{\partial x} \wedge \frac{\partial}{\partial y} \wedge \alpha\left(z \frac{\partial}{\partial z}+u \frac{\partial}{\partial u}\right), \quad \alpha \neq 0$,

(v) $\eta_{5}=\frac{\partial}{\partial x} \wedge \frac{\partial}{\partial y} \wedge \beta\left(u \frac{\partial}{\partial z}-z \frac{\partial}{\partial u}\right), \beta \neq 0$,

(vi) $\eta_{6}=\frac{\partial}{\partial x} \wedge \frac{\partial}{\partial y} \wedge(\alpha z) \frac{\partial}{\partial z}, \quad \alpha \neq 0$,

(vii) $\eta_{7}=\frac{\partial}{\partial x} \wedge \frac{\partial}{\partial y} \wedge u \frac{\partial}{\partial z}$.

A linear Nambu-Poisson tensor of type (I) is one of exact Nambu-Poisson tensors. And this case was already considered in the previous section. Hence in this section we will only give the results concerning nondegenerate NambuPoisson tensors (i.e. $f= \pm x^{2} \pm y^{2} \pm z^{2} \pm u^{2}$ ) for type (I). And here we will mainly study the computation for type (II).

Throughout this section, we will use the following notations:

- $\mathcal{F}$ is the algebra of real-valued $C^{\infty}$ functions on $\mathbb{R}^{4}(x, y, z, u)$;

- $\tilde{\mathcal{G}}$ is the algebra of real-valued $C^{\infty}$ functions on $\mathbb{R}^{3}(y, z, u)$;

- $\tilde{\mathcal{F}}$ is the algebra of real-valued $C^{\infty}$ functions on $\mathbb{R}^{2}(z, u)$;

- $\mathcal{F}(1)$ is the algebra of formal functions of one variable;

- $\chi\left(\mathbb{R}^{4}\right)$ is the Lie algebra of all vector fields on $\mathbb{R}^{4}$;

- $\Omega^{k}$ is the space of $k$-forms on $\mathbb{R}^{4}$. 


\section{§5.2. Computation of Nambu-Poisson cohomology of type (I)}

In this subsection, we confine ourselves to nondegenerate linear NambuPoisson tensors of type (I). This means that $f= \pm x^{2} \pm y^{2} \pm z^{2} \pm u^{2}$ and it is clear that $f$ is of finite codimension. We get the following results by using Theorem 4.1 of C. A. Roche [13]. We use the same notations as those of the previous section. Let $\eta$ be a linear Nambu-Poisson tensor of type (I) defined by $i(\eta) \Omega=d f$. Then we get the following flat relative cohomology. In Table 1 , $D^{i}$ denotes an $i$-dimensional ball.

Table 1. Flat Relative Cohomology

\begin{tabular}{c|c|c|c|c|c|c}
$f$ & $X_{+c}$ & $X_{-c}$ & $H_{\infty \text { rel }}^{0}$ & $H_{\infty \text { rel }}^{1}$ & $H_{\infty \text { rel }}^{2}$ & $H_{\infty \text { rel }}^{3}$ \\
\hline$x^{2}+y^{2}+z^{2}+u^{2}$ & $S^{3}$ & $\phi$ & $m_{+}^{\infty}$ & 0 & 0 & $m_{+}^{\infty}$ \\
\hline$x^{2}+y^{2}+z^{2}-u^{2}$ & $S^{2} \times D^{1}$ & $S^{0} \times D^{3}$ & $m_{+}^{\infty} \times m_{-}^{\infty} \times m_{-}^{\infty}$ & 0 & $m_{+}^{\infty}$ & 0 \\
\hline$x^{2}+y^{2}-z^{2}-u^{2}$ & $S^{1} \times D^{2}$ & $S^{1} \times D^{2}$ & $m_{+}^{\infty} \times m_{-}^{\infty}$ & $m_{+}^{\infty} \times m_{-}^{\infty}$ & 0 & 0 \\
\hline$x^{2}-y^{2}-z^{2}-u^{2}$ & $S^{0} \times D^{3}$ & $S^{2} \times D^{1}$ & $m_{+}^{\infty} \times m_{+}^{\infty} \times m_{-}^{\infty}$ & 0 & $m_{-}^{\infty}$ & 0 \\
\hline$-x^{2}-y^{2}-z^{2}-u^{2}$ & $\phi$ & $S^{3}$ & $m_{-}^{\infty}$ & 0 & 0 & $m_{-}^{\infty}$
\end{tabular}

Combining the results in Table 1 with Theorem 4.2 and Theorem 4.6, we can compute cohomology of type (I). In computing $H_{N P}^{3}$, note that $\Omega^{4} / d f \wedge \Omega^{3} \cong$ $\mathbb{R}$, and $\mu=1$. We collect the results in the following table.

Table 2. Exact Nambu-Poisson Cohomology

\begin{tabular}{c|c|c|c|c}
$f$ & $H_{N P}^{0}$ & $H_{N P}^{1}$ & $H_{N P}^{2}$ & $H_{N P}^{3}$ \\
\hline$x^{2}+y^{2}+z^{2}+u^{2}$ & $C^{\infty}\left(\mathbb{R}^{+}\right)$ & 0 & 0 & $C^{\infty}\left(\mathbb{R}^{+}\right) \oplus \mathbb{R}$ \\
\hline$x^{2}+y^{2}+z^{2}-u^{2}$ & $C^{\infty}\left(\mathbb{R}^{+}\right) \times C^{\infty}\left(\mathbb{R}^{-}\right) \times C^{\infty}\left(\mathbb{R}^{-}\right)$ & 0 & $m_{+}^{\infty}$ & $\mathcal{F}(1) \oplus \mathbb{R}$ \\
\hline$x^{2}+y^{2}-z^{2}-u^{2}$ & $C^{\infty}\left(\mathbb{R}^{+}\right) \times C^{\infty}\left(\mathbb{R}^{-}\right)$ & $m_{+}^{\infty} \times m_{-}^{\infty}$ & 0 & $\mathcal{F}(1) \oplus \mathbb{R}$ \\
\hline$x^{2}-y^{2}-z^{2}-u^{2}$ & $C^{\infty}\left(\mathbb{R}^{+}\right) \times C^{\infty}\left(\mathbb{R}^{+}\right) \times C^{\infty}\left(\mathbb{R}^{-}\right)$ & 0 & $m_{-}^{\infty}$ & $\mathcal{F}(1) \oplus \mathbb{R}$ \\
\hline$-x^{2}-y^{2}-z^{2}-u^{2}$ & $C^{\infty}\left(\mathbb{R}^{-}\right)$ & 0 & 0 & $C^{\infty}\left(\mathbb{R}^{-}\right) \oplus \mathbb{R}$
\end{tabular}

\section{§5.3. Computation of Nambu-Poisson cohomology of type (II)}

In this subsection, we compute Nambu-Poisson cohomology of type (II). Denote by $\mathfrak{g}_{i}$ the Lie algebra corresponding to $\eta_{i}, \quad i=1,2, \ldots, 7$. Recall that $\mathfrak{g}_{i}$ is defined by $\mathfrak{g}_{i}=i\left(\Omega^{2}\right) \eta_{i}$. Then each $\mathfrak{g}_{i}$ is spanned over $\mathcal{F}$ by several vector 
fields as follows.

$$
\begin{aligned}
\mathfrak{g}_{1} & =\left\langle z \frac{\partial}{\partial x}, u \frac{\partial}{\partial x}, z \frac{\partial}{\partial y}, u \frac{\partial}{\partial y},(\alpha z+u) \frac{\partial}{\partial z}+\alpha u \frac{\partial}{\partial u}\right\rangle ; \\
\mathfrak{g}_{2} & =\left\langle z \frac{\partial}{\partial x}, u \frac{\partial}{\partial x}, z \frac{\partial}{\partial y}, u \frac{\partial}{\partial y}, \alpha z \frac{\partial}{\partial z}+\beta u \frac{\partial}{\partial u}\right\rangle ; \\
\mathfrak{g}_{3} & =\left\langle z \frac{\partial}{\partial x}, u \frac{\partial}{\partial x}, z \frac{\partial}{\partial y}, u \frac{\partial}{\partial y},(\alpha z-\beta u) \frac{\partial}{\partial z}+(\beta z+\alpha u) \frac{\partial}{\partial u}\right\rangle ; \\
\mathfrak{g}_{4} & =\left\langle z \frac{\partial}{\partial x}, u \frac{\partial}{\partial x}, z \frac{\partial}{\partial y}, u \frac{\partial}{\partial y}, z \frac{\partial}{\partial z}+u \frac{\partial}{\partial u}\right\rangle ; \\
\mathfrak{g}_{5} & =\left\langle z \frac{\partial}{\partial x}, u \frac{\partial}{\partial x}, z \frac{\partial}{\partial y}, u \frac{\partial}{\partial y}, u \frac{\partial}{\partial z}-z \frac{\partial}{\partial u}\right\rangle ; \\
\mathfrak{g}_{6} & =\left\langle z \frac{\partial}{\partial x}, z \frac{\partial}{\partial y}, z \frac{\partial}{\partial z}\right\rangle ; \\
\mathfrak{g}_{7} & =\left\langle u \frac{\partial}{\partial x}, u \frac{\partial}{\partial y}, u \frac{\partial}{\partial z}\right\rangle .
\end{aligned}
$$

As is easily seen, we know that

$$
\Lambda^{4} \mathfrak{g}_{i}=0, \text { for } 1 \leq i \leq 7
$$

Denote by $H_{N P}^{k}\left(\eta_{i}\right)$ the $k$-th cohomology group corresponding to the Nambu-Poisson tensor $\eta_{i}$. Then for $1 \leq i \leq 7, H_{N P}^{k}\left(\eta_{i}\right)=0$ if $4 \leq k$.

For $0 \leq k \leq 4, I^{k} \subset \Omega^{k}$ is similarly defined as in the previous section (see Definition 4.2). Then we also have $C^{k} \cong \Omega^{k} / I^{k}$. First let us determine explicit forms of all $I^{k}$. They are summarized in the following lemma.

Lemma 5.1. Let $A, B, C, D, E, F$ be elements of $\mathcal{F}$.

(a) In case of $\eta_{1}$,

$$
\begin{aligned}
I^{1}= & \{C d z+D d u \mid(\alpha z+u) C+\alpha u D=0\} \\
I^{2}= & \{B d x \wedge d z+C d x \wedge d u+D d y \wedge d z+E d y \wedge d u \\
& +F d z \wedge d u \mid(\alpha z+u) B+\alpha u C=0,(\alpha z+u) D+\alpha u E=0\} \\
I^{3}= & \{A d x \wedge d y \wedge d z+B d x \wedge d y \wedge d u+C d x \wedge d z \wedge d u \\
& +D d y \wedge d z \wedge d u \mid(\alpha z+u) A+\alpha u B=0\} \\
I^{4}= & \Omega^{4}
\end{aligned}
$$


(b) In case of $\eta_{2}$,

$$
\begin{aligned}
I^{1}= & \{C d z+D d u \mid \alpha z C+\beta u D\}, \\
I^{2}= & \{B d x \wedge d z+C d x \wedge d u+D d y \wedge d z+E d y \wedge d u \\
& +F d z \wedge d u \mid \alpha z B+\beta u C=0, \alpha z D+\beta u E=0\}, \\
I^{3}= & \{A d x \wedge d y \wedge d z+B d x \wedge d y \wedge d u+C d x \wedge d z \wedge d u \\
& +D d y \wedge d z \wedge d u \mid \alpha z A+\beta u B=0\}, \\
I^{4}= & \Omega^{4} .
\end{aligned}
$$

(c) In case of $\eta_{3}$,

$$
\begin{aligned}
I^{1}= & \{C d z+D d u \mid(\alpha z-\beta u) C+(\beta z+\alpha u) D=0\}, \\
I^{2}= & \{B d x \wedge d z+C d x \wedge d u+D d y \wedge d z+E d y \wedge d u \\
& +F d z \wedge d u \mid(\alpha z-\beta u) B+(\beta z+\alpha u) C=0, \\
(\alpha z-\beta u) D+(\beta z+\alpha u) E=0\}, & \\
I^{3}= & \{A d x \wedge d y \wedge d z+B d x \wedge d y \wedge d u+C d x \wedge d z \wedge d u \\
& +D d y \wedge d z \wedge d u \mid(\alpha z-\beta u) A+(\beta z+\alpha u) B=0\}, \\
I^{4}= & \Omega^{4} .
\end{aligned}
$$

(d) In case of $\eta_{4}$,

$$
\begin{aligned}
I^{1}= & \{C d z+D d u \mid z C+u D=0\}, \\
I^{2}= & \{B d x \wedge d z+C d x \wedge d u+D d y \wedge d z+E d y \wedge d u \\
& +F d z \wedge d u \mid z B+u C=0, z D+u E=0\}, \\
I^{3}= & \{A d x \wedge d y \wedge d z+B d x \wedge d y \wedge d u+C d x \wedge d z \wedge d u \\
& +D d y \wedge d z \wedge d u \mid z A+u B=0\}, \\
I^{4}= & \Omega^{4} .
\end{aligned}
$$

(e) In case of $\eta_{5}$,

$$
\begin{aligned}
I^{1}= & \{C d z+D d u \mid z D-u C=0\} \\
I^{2}= & \{B d x \wedge d z+C d x \wedge d u+D d y \wedge d z+E d y \wedge d u \\
& +F d z \wedge d u \mid u B-z C=0, u D-z E=0\}, \\
I^{3}= & \{A d x \wedge d y \wedge d z+B d x \wedge d y \wedge d u+C d x \wedge d z \wedge d u \\
& +D d y \wedge d z \wedge d u \mid u A-z B=0\}, \\
I^{4}= & \Omega^{4} .
\end{aligned}
$$


(f) In cases of $\eta_{6}$ and $\eta_{7}$,

$$
\begin{aligned}
I^{1} & =\mathcal{F} d u \\
I^{2} & =\mathcal{F} d x \wedge d u+\mathcal{F} d y \wedge d u+\mathcal{F} d z \wedge d u \\
I^{3} & =\mathcal{F} d x \wedge d y \wedge d u+\mathcal{F} d x \wedge d z \wedge d u+\mathcal{F} d y \wedge d z \wedge d u \\
I^{4} & =\Omega^{4}
\end{aligned}
$$

Proof. Straightforward computation.

In linear cases, we also have the following commutative diagram which is similar to that of relative cases. (Its proof is obtained as a direct consequence of the definition of the operator $\partial$.)

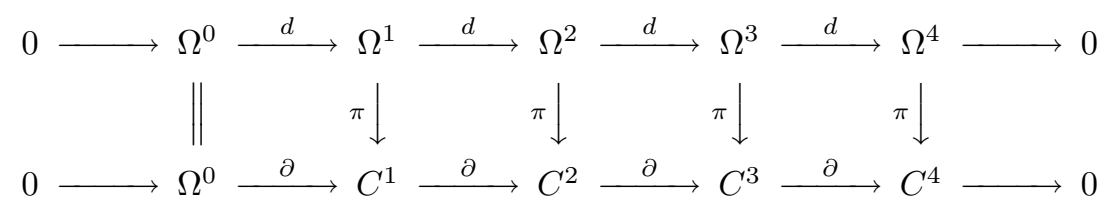

Now let us compute Nambu-Poisson cohomology for Nambu-Poisson tensors $\eta_{i}, 1 \leq i \leq 7$. Recall that $I^{4}=\Omega^{4}$. This means that $C^{4}=0$ in the above commutative diagram. Hence we have only to compute $H_{N P}^{k}\left(\eta_{i}\right)$ for $0 \leq k \leq 3$.

We denote by $Z^{k}$ the space of cocycles and by $B^{k}$ the space of coboundaries in $C^{k}$. Clearly it holds that $B^{k} \subset Z^{k} \subset C^{k}$, and by definition, $H_{N P}^{k}=Z^{k} / B^{k}$.

Definition 5.1. We define the subspaces $\tilde{Z}^{k}$ and $\tilde{B}^{k}$ of $\Omega^{k}$ as follows.

$$
\begin{aligned}
& \tilde{Z}^{k}=\left\{c \in \Omega^{k} \mid d c \in I^{k+1}\right\}, \\
& \tilde{B}^{k}=d \Omega^{k-1} .
\end{aligned}
$$

Note that it holds $I^{k} \subset \tilde{Z}^{k}$.

Proposition 5.2. $\quad H_{N P}^{k} \cong \tilde{Z}^{k} /\left(\tilde{B}^{k}+I^{k}\right)$ for $1 \leq k \leq 3$.

Proof. We first prove that $\pi^{-1}\left(Z^{k}\right)=\tilde{Z}^{k}$. For $c \in \pi^{-1}\left(Z^{k}\right)$, we have $0=\partial(\pi c)=\pi(d c)$. Hence $d c \in I^{k+1}$ and this implies $c \in \tilde{Z}^{k}$. The converse is clear. Hence the linear mapping $\pi: \tilde{Z}^{k} \longrightarrow Z^{k}$ is surjective. Since $\operatorname{ker} \pi=I^{k}$, we have $Z^{k} \cong \tilde{Z}^{k} / I^{k}$. Next note that $B^{k}=\partial C^{k-1}=\partial\left(\pi \Omega^{k-1}\right)=\pi\left(d \Omega^{k-1}\right)=$ $\pi \tilde{B}^{k}$. Hence $\pi^{-1}\left(B^{k}\right)=\tilde{B}^{k}+I^{k}$, and $B^{k} \cong\left(\tilde{B}^{k}+I^{k}\right) / I^{k}$. Finally we have

$$
H_{N P}^{k}=Z^{k} / B^{k} \cong\left(\tilde{Z}^{k} / I^{k}\right) /\left(\left(\tilde{B}^{k}+I^{k}\right) / I^{k}\right) \cong \tilde{Z}^{k} /\left(\tilde{B}^{k}+I^{k}\right) .
$$


To compute Nambu-Poisson cohomology for linear Nambu-Poisson tensors, the following lemma is useful. After the preparation of this paper, T. Fukuda informed me that J. Mather [8] and T. Fukuda and S. Janeczko [4] had already proved an analogous kind of result in a more general situation. So we omit the proof.

Lemma 5.3. Let $f(x, y, z, u)$ and $g(x, y, z, u)$ be $C^{\infty}$-functions on $\mathbb{R}^{4}$ $(x, y, z, u)$, and let $A(z, u)$ and $B(z, u)$ be linear functions of two variables $z, u$ such that $\partial(A, B) / \partial(z, u) \neq 0$. If $f(x, y, z, u)$ and $g(x, y, z, u)$ satisfy the condition:

$$
A(z, u) \cdot f(x, y, z, u)=B(z, u) \cdot g(x, y, z, u),
$$

then there exists a function $h(x, y, z, u) \in C^{\infty}\left(\mathbb{R}^{4}\right)$ such that

$$
\left\{\begin{array}{c}
f(x, y, z, u)=B(z, u) \cdot h(x, y, z, u), \\
g(x, y, z, u)=A(z, u) \cdot h(x, y, z, u) .
\end{array}\right.
$$

Let us begin with computing Nambu-Poisson cohomology for NambuPoisson tensor $\eta_{1}=\frac{\partial}{\partial x} \wedge \frac{\partial}{\partial y} \wedge\left\{(\alpha z+u) \frac{\partial}{\partial z}+\alpha u \frac{\partial}{\partial u}\right\}$, where $\alpha \neq 0$. Then the corresponding Lie algebra $\mathfrak{g}_{1}$ is spanned by $\left\langle z \frac{\partial}{\partial x}, u \frac{\partial}{\partial x}, z \frac{\partial}{\partial y}, u \frac{\partial}{\partial y},(\alpha z+u) \frac{\partial}{\partial z}+\alpha u \frac{\partial}{\partial u}\right\rangle$ over $\mathcal{F}$. It is clear that $H_{N P}^{k}\left(\eta_{1}\right)=0$ for $k \geq 4$ since $\Lambda^{4} \mathfrak{g}_{1}=0$.

\section{Lemma 5.4.}

(a) Put $c=A d x+B d y+C d z+D d u$. Then $c \in \tilde{Z}^{1}$ if and only if

$$
\left\{\begin{aligned}
B_{x} & =A_{y} \\
(\alpha z+u)\left(C_{x}-A_{z}\right) & =\alpha u\left(A_{u}-D_{x}\right), \\
(\alpha z+u)\left(C_{y}-B_{z}\right) & =\alpha u\left(B_{u}-D_{y}\right) .
\end{aligned}\right.
$$

(b) Put $c=A d x \wedge d y+B d x \wedge d z+C d x \wedge d u+D d y \wedge d z+E d y \wedge d u+F d z \wedge d u$. Then $c \in \tilde{Z}^{2}$ if and only if $(\alpha z+u)\left(A_{z}-B_{y}+D_{x}\right)=-\alpha u\left(A_{u}-C_{y}+E_{x}\right)$.

(c) $\tilde{Z}^{3}=\Omega^{3}$.

Proof. We have only to recall that $c \in \tilde{Z}^{k}$ if and only if $d c \in I^{k+1}$. Then direct computation shows the above results. 
Theorem 5.5. Let $\eta_{1}=\frac{\partial}{\partial x} \wedge \frac{\partial}{\partial y} \wedge\left\{(\alpha z+u) \frac{\partial}{\partial z}+\alpha u \frac{\partial}{\partial u}\right\}$. Then we have

$$
\begin{aligned}
& H_{N P}^{0}\left(\eta_{1}\right) \cong \mathbb{R}, \\
& H_{N P}^{1}\left(\eta_{1}\right) \cong \tilde{\mathcal{F}} / \tilde{\mathcal{F}}_{1} \cong \mathcal{I}_{\mathbb{R}^{2}} / \tilde{\mathcal{F}}_{1} \cap \mathcal{I}_{\mathbb{R}^{2}}, \\
& \quad \text { where } \tilde{\mathcal{F}}_{1}=\left\{(\alpha z+u) \tilde{h}_{z}+\alpha u \tilde{h}_{u}+2 \alpha \tilde{h} \mid \tilde{h} \in \tilde{\mathcal{F}}\right\} \\
& H_{N P}^{2}\left(\eta_{1}\right) \cong \tilde{\mathcal{G}} / \tilde{\mathcal{G}}_{1} \cong \tilde{\mathcal{I}}_{\mathbb{R}^{3}} / \tilde{\mathcal{G}}_{1} \cap \tilde{\mathcal{I}}_{\mathbb{R}^{3}} \\
& \quad \text { where } \tilde{\mathcal{G}}_{1}=\left\{(\alpha z+u) \tilde{g}_{z}+\alpha u \tilde{g}_{u}+2 \alpha \tilde{g} \mid \tilde{g} \in \tilde{\mathcal{G}}\right\} \\
& H_{N P}^{k}\left(\eta_{1}\right)=0 \text { for } k \geq 3
\end{aligned}
$$

In the above results, $\tilde{\mathcal{I}}_{\mathbb{R}^{2}}$ (resp. $\tilde{\mathcal{I}}_{\mathbb{R}^{3}}$ ) stands for the space of functions defined on $\mathbb{R}^{2}(z, u)$ (resp. $\left.\mathbb{R}^{3}(y, z, u)\right)$ which are flat at the origin.

Proof. Let $f$ be an element of $H_{N P}^{0}\left(\eta_{1}\right)$. Then $f=f(z, u)$, and it holds that $(\alpha z+u) f_{z}+\alpha u f_{u}=0$. The solution is $f(z, u)=\phi\left(\frac{\alpha z-u \log u}{u}\right)$, where $\phi$ is any $C^{\infty}$-function of 1 -variable. Hence smooth solutions $f(z, u)$ are only constants.

For the computation of $H_{N P}^{1}\left(\eta_{1}\right)$, put $c=A d x+B d y+C d z+D d u \in \tilde{Z}^{1}$. Then by Lemma 5.4(a), there exists a function $h \in \mathcal{F}$ such that $A=h_{x}, B=h_{y}$. Then the last two equations in (a) can be rewritten as follows.

$$
\begin{aligned}
& \alpha u\left(h_{u}-D\right)_{x}=(\alpha z+u)\left(C-h_{z}\right)_{x}, \\
& \alpha u\left(h_{u}-D\right)_{y}=(\alpha z+u)\left(C-h_{z}\right)_{y} .
\end{aligned}
$$

Hence by Lemma 5.3, there exist $k, l \in \mathcal{F}$, such that $\left(C-h_{z}\right)_{x}=$ $\alpha u k,\left(h_{u}-D\right)_{x}=(\alpha z+u) k,\left(C-h_{z}\right)_{y}=\alpha u l, \quad\left(h_{u}-D\right)_{y}=(\alpha z+u) l$. Then we have

$$
\begin{aligned}
& C-h_{z}=\alpha u \int k d x+\phi_{1}(y, z, u)=\alpha u \int l d y+\phi_{2}(x, z, u), \\
& h_{u}-D=(\alpha z+u) \int k d x+\psi_{1}(y, z, u)=(\alpha z+u) \int l d y+\psi_{2}(x, z, u) .
\end{aligned}
$$

By the integrability condition, it holds that $k_{y}=l_{x}$. And we have $(C-$ $\left.h_{z}\right)_{y}=\alpha u \int k_{y} d x+\left(\phi_{1}\right)_{y}=\alpha u \int l_{x} d x+\left(\phi_{1}\right)_{y}=\alpha u\left(l-\bar{\phi}_{1}(y, z, u)\right)+\left(\phi_{1}\right)_{y}$ for some function $\bar{\phi}_{1}(y, z, u)$. On the other hand, since $\left(C-h_{z}\right)_{y}=\alpha u l$, we must have $\left(\phi_{1}\right)_{y}=\alpha u \bar{\phi}_{1}(y, z, u)$, and hence $\phi_{1}(y, z, u)=\alpha u \int \bar{\phi}_{1}(y, z, u) d y+\tilde{\phi}_{1}(z, u)$ for some function $\tilde{\phi}_{1}(z, u)$. By the same way as above, we have $\left(h_{u}-D\right)_{y}=$ $(\alpha z+u) \int k_{y} d x+\left(\psi_{1}\right)_{y}=(\alpha z+u) \int l_{x} d x+\left(\psi_{1}\right)_{y}=(\alpha z+u)\left(l-\bar{\psi}_{1}(y, z, u)\right)+$ $\left(\psi_{1}\right)_{y}=(\alpha z+u) l$. Hence we have $\psi_{1}(y, z, u)=(\alpha z+u) \int \bar{\psi}_{1} d y+\tilde{\psi}_{1}(z, u)$ 
for some functions $\bar{\psi}_{1}(y, z, u)$ and $\tilde{\psi}_{1}(z, u)$. Now $C$ and $D$ can be written as follows.

$$
\begin{aligned}
& C=h_{z}+\alpha u \int k d x+\alpha u \int \bar{\phi}_{1} d y+\tilde{\phi}_{1}(z, u), \\
& D=h_{u}-(\alpha z+u) \int k d x-(\alpha z+u) \int \bar{\psi}_{1} d y-\tilde{\psi}_{1}(z, u) .
\end{aligned}
$$

Then we have

$$
\begin{aligned}
\alpha u\left(B_{u}-D_{y}\right) & =\alpha u\left(h_{y u}+(\alpha z+u) \int k_{y} d x+(\alpha z+u) \bar{\psi}_{1}-h_{y u}\right) \\
& =\alpha u(\alpha z+u)\left(\int k_{y} d x+\overline{\psi_{1}}\right) \\
(\alpha z+u)\left(C_{y}-B_{z}\right) & =(\alpha z+u)\left(h_{y z}+\alpha u \int k_{y} d x+\alpha u \bar{\phi}_{1}-h_{y z}\right) \\
& =\alpha u(\alpha z+u)\left(\int k_{y} d x+\bar{\phi}_{1}\right) .
\end{aligned}
$$

Since $\alpha u\left(B_{u}-D_{y}\right)=(\alpha z+u)\left(C_{y}-B_{z}\right)$, we get $\bar{\phi}_{1}=\bar{\psi}_{1}$. Thus $c \in \tilde{Z}^{1}$ has the following expression.

$$
\begin{aligned}
c= & A d x+B d y+C d z+D d u \\
= & h_{x} d x+h_{y} d y+\left(h_{z}+\alpha u \int k d x+\alpha u \int \bar{\phi}_{1}(y, z, u) d y+\tilde{\phi}_{1}(z, u)\right) d z \\
& +\left(h_{u}-(\alpha z+u) \int k d x-(\alpha z+u) \int \bar{\psi}_{1}(y, z, u) d y-\tilde{\psi}_{1}(z, u)\right) d u \\
= & d h+\alpha u\left(\int k d x+\int \bar{\phi}_{1} d y\right) d z+\tilde{\phi}_{1}(z, u) d z \\
& -(\alpha z+u)\left(\int k d x+\int \bar{\phi}_{1} d y\right) d u-\tilde{\psi}_{1}(z, u) d u .
\end{aligned}
$$

Note that $d h+\alpha u\left(\int k d x+\int \bar{\phi}_{1} d y\right) d z-(\alpha z+u)\left(\int k d x+\int \bar{\phi}_{1} d y\right) d u$ is contained in $\tilde{B}^{1}+I^{1}$. Hence by Proposition 5.2, we can consider $H_{N P}^{1}\left(\eta_{1}\right)$ as $\left\{\tilde{\phi}_{1}(z, u) d z-\tilde{\psi}_{1}(z, u) d u \mid \tilde{\phi}_{1}, \tilde{\psi}_{1} \in \tilde{\mathcal{F}}\right\}$ modulo $\tilde{B}^{1}+I^{1}$. Let $A_{1}$ be the space of 1 -forms on $\mathbb{R}^{2}(z, u), A_{2}$ be the space of 2 -forms on $\mathbb{R}^{2}(z, u)$, and $B_{1}$ be the space of exact 1 -forms on $\mathbb{R}^{2}(z, u)$. It is clear that $A_{1} / B_{1} \cong A_{2} \cong \tilde{\mathcal{F}}$. We also define the subspace $C_{1}$ of $A_{1}$ by

$$
C_{1}=\{\alpha u \tilde{h} d z-(\alpha z+u) \tilde{h} d u \mid \tilde{h} \in \tilde{\mathcal{F}}\} .
$$

Note that $B_{1} \subset \tilde{B}^{1}$ and $C_{1} \subset I^{1}$. Then we have 


$$
\begin{aligned}
H_{N P}^{1}\left(\eta_{1}\right) & \cong A_{1} /\left(B_{1}+C_{1}\right) \\
& \cong\left(A_{1} / B_{1}\right) /\left(\left(B_{1}+C_{1}\right) / B_{1}\right) \\
& \cong\left(A_{1} / B_{1}\right) /\left(C_{1} / B_{1} \cap C_{1}\right) .
\end{aligned}
$$

Let $\alpha u \tilde{h} d z-(\alpha z+u) \tilde{h} d u$ be any element of $B_{1} \cap C_{1}$. Then $\tilde{h}$ has the form $(\alpha z+u) \tilde{h}_{z}+\alpha u \tilde{h}_{u}=-2 \alpha \tilde{h}$. Any solution has the form $\tilde{h}=u^{-2} \phi\left(\frac{\alpha z-u \log u}{u}\right)$ with an arbitrary function $\phi$ of 1 -variable. Hence $C^{\infty}$-solution is only $\tilde{h}=0$, and $B_{1} \cap C_{1}=0$. This means that $C_{1} \cong d C_{1}$, and we know that $C_{1}$ is isomorphic to the space

$$
\tilde{\mathcal{F}}_{1}=\left\{(\alpha z+u) \tilde{h}_{z}+\alpha u \tilde{h}_{u}+2 \alpha \tilde{h} \mid \tilde{h} \in \tilde{\mathcal{F}}\right\} .
$$

Let $F$ be the space of formal functions on $\mathbb{R}^{2}(z, u)$. Each element $[\tilde{h}]$ of $F$ is obtained by the formal Taylor expansion of $\tilde{h} \in \tilde{\mathcal{F}}$ at the origin. It is easy to see that the mapping $T: \tilde{h} \longrightarrow[\tilde{h}]$ is linear and surjective. The kernel of $T$ will be denoted by $\mathcal{I}_{\mathbb{R}^{2}}$. Put

$$
[\tilde{h}]=\sum_{i, j \geq 0} a_{i j} z^{i} u^{j} \in F .
$$

Then we have

$$
\begin{aligned}
& {\left[(\alpha z+u) \tilde{h}_{z}+\alpha u \tilde{h}_{u}+2 \alpha \tilde{h}\right]} \\
& =\sum_{i, j \geq 0} \alpha(i+j+2) a_{i j} z^{i} u^{j} \\
& +\sum_{i \geq 0, j \geq 1}(i+1) a_{i+1, j-1} z^{i} u^{j}
\end{aligned}
$$

and we know that $T(\tilde{\mathcal{F}})=T\left(\tilde{\mathcal{F}}_{1}\right)=F$. This means that for any $f \in \tilde{\mathcal{F}}$, there exists $g \in \tilde{\mathcal{F}}_{1}$ such that $f-g \in \mathcal{I}_{\mathbb{R}^{2}}$, and it holds that $\tilde{\mathcal{F}}=\tilde{\mathcal{F}}_{1}+\mathcal{I}_{\mathbb{R}^{2}}$. Thus we obtain that

$$
H_{N P}^{1}\left(\eta_{1}\right) \cong\left(A_{1} / B_{1}\right) / C_{1} \cong \tilde{\mathcal{F}} / \tilde{\mathcal{F}}_{1} \cong \mathcal{I}_{\mathbb{R}^{2}} / \tilde{\mathcal{F}}_{1} \cap \mathcal{I}_{\mathbb{R}^{2}}
$$

For the computation of $H_{N P}^{2}\left(\eta_{1}\right)$, let $\gamma=A d x \wedge d y+B d x \wedge d z+C d x \wedge$ $d u+D d y \wedge d z+E d y \wedge d u+F d z \wedge d u$ be any element of $\tilde{Z}^{2}$. Then by Lemma 5.3 and by Lemma $5.4(\mathrm{~b})$, there exists a function $k(x, y, z, u) \in \mathcal{F}$ such that

$$
\begin{aligned}
& A_{z}-B_{y}+D_{x}=\alpha u k, \\
& A_{u}-C_{y}+E_{x}=-(\alpha z+u) k .
\end{aligned}
$$

Then there exist two functions $\phi_{1}$ and $\phi_{2}$ of $\tilde{\mathcal{G}}$ such that $D$ and $E$ have the following expressions. 


$$
\begin{aligned}
& D=\alpha u \int k d x+\int B_{y} d x-\int A_{z} d x+\phi_{1}(y, z, u), \\
& E=-(\alpha z+u) \int k d x+\int C_{y} d x-\int A_{u} d x+\phi_{2}(y, z, u) .
\end{aligned}
$$

Define a 1 -form $\varpi$ by $\varpi=P d x+Q d y+R d z+S d u$. If we put $Q=\int A d x, R=$ $\int B d x, S=\int C d x$, then

$$
\begin{aligned}
d \varpi= & \left(A-P_{y}\right) d x \wedge d y+\left(B-P_{z}\right) d x \wedge d z+\left(C-P_{u}\right) d x \wedge d u \\
& +\left(\int B_{y} d x-\int A_{z} d x\right) d y \wedge d z+\left(\int C_{y} d x-\int A_{u} d x\right) d y \wedge d u \\
& +\left(\int C_{z} d x-\int B_{u} d x\right) d z \wedge d u .
\end{aligned}
$$

Thus we have

$$
\begin{aligned}
\gamma= & d \varpi+d(x \cdot d P)+\left(\alpha u \int k d x\right) d y \wedge d z+\left(-(\alpha z+u) \int k d x\right) d y \wedge d u \\
& +\left(F+\int B_{u} d x-\int C_{z} d x\right) d z \wedge d u+\phi_{1} d y \wedge d z+\phi_{2} d y \wedge d u .
\end{aligned}
$$

The first five terms of $\gamma$ belong to $\tilde{B}^{2}+I^{2}$. It will be denoted by $B I$. Then $\gamma=B I+\phi_{1} d y \wedge d z+\phi_{2} d y \wedge d u$. By Proposition 5.2, we can consider $H_{N P}^{2}\left(\eta_{1}\right)$ as $\left\{\phi_{1}(y, z, u) d y \wedge d z+\phi_{2}(y, z, u) d y \wedge d u \mid \phi_{1}, \phi_{2} \in \tilde{\mathcal{G}}\right\}$ modulo $\tilde{B}^{2}+I^{2}$. Let us define some subspaces of the space of 2 -forms on $\mathbb{R}^{3}(y, z, u)$ as follows.

$$
\begin{aligned}
U_{2} & =\left\{\phi_{1} d y \wedge d z+\phi_{2} d y \wedge d u \mid \phi_{1}, \phi_{2} \in \tilde{\mathcal{G}}\right\}, \\
V_{2} & =\left\{\phi_{1} d y \wedge d z+\phi_{2} d y \wedge d u \in U_{2} \mid\left(\phi_{1}\right)_{u}=\left(\phi_{2}\right)_{z}\right\}, \\
W_{2} & =\{\alpha u \tilde{g} d y \wedge d z-(\alpha z+u) \tilde{g} d y \wedge d u \mid \tilde{g} \in \tilde{\mathcal{G}}\} .
\end{aligned}
$$

Moreover put

$$
U_{3}=\{\tilde{h} d y \wedge d z \wedge d u \mid \tilde{h} \in \tilde{\mathcal{G}}\} .
$$

Since $d U_{2}=U_{3}$, we know that $U_{2} / V_{2} \cong U_{3} \cong \tilde{\mathcal{G}}$. Note that $V_{2} \subset \tilde{B}^{2}$ and $W_{2} \subset I^{2}$. Then we have

$$
\begin{aligned}
H_{N P}^{2}\left(\eta_{1}\right) & \cong U_{2} /\left(V_{2}+W_{2}\right) \\
& \cong\left(U_{2} / V_{2}\right) /\left(\left(V_{2}+W_{2}\right) / V_{2}\right) \\
& \cong\left(U_{2} / V_{2}\right) /\left(W_{2} / V_{2} \cap W_{2}\right) .
\end{aligned}
$$

Let $\alpha u \tilde{g} d y \wedge d z-(\alpha z+u) \tilde{g} d y \wedge d u$ be any element of $V_{2} \cap W_{2}$. Then $\tilde{g}$ must satisfy the equation $(\alpha z+u) \tilde{g}_{z}+\alpha u \tilde{g}_{u}=-2 \alpha \tilde{g}$. Any solution of this equation has the form $\tilde{g}(y, z, u)=u^{-2} \psi\left(\frac{\alpha z-u \log u}{u}, y\right)$, where $\psi$ is any function of 2 -variables. 
Hence $C^{\infty}$-solution is only $\tilde{g}=0$. This means that $V_{2} \cap W_{2}=0$. We define a subspace $\tilde{\mathcal{G}}_{1}$ of $\tilde{\mathcal{G}}$ by

$$
\tilde{\mathcal{G}}_{1}=\left\{(\alpha z+u) \tilde{g}_{z}+\alpha u \tilde{g}_{u}+2 \alpha \tilde{g} \mid \tilde{g} \in \tilde{\mathcal{G}}\right\} .
$$

Then it is clear that $W_{2} / V_{2} \cap W_{2}=W_{2} \cong \tilde{\mathcal{G}}_{1}$. Let $\mathcal{I}_{\mathbb{R}^{3}}$ be the space of flat functions at the origin defined on $\mathbb{R}^{3}(y, z, u)$. By the analogous consideration as the case of $H_{N P}^{1}\left(\eta_{1}\right)$, we obtain

$$
H_{N P}^{2}\left(\eta_{1}\right) \cong \tilde{\mathcal{G}} / \tilde{\mathcal{G}}_{1} \cong \mathcal{I}_{\mathbb{R}^{3}} / \tilde{\mathcal{G}}_{1} \cap \mathcal{I}_{\mathbb{R}^{3}} .
$$

For the computation of $H_{N P}^{3}\left(\eta_{1}\right)$, let $\delta=A d x \wedge d y \wedge d z+B d x \wedge d y \wedge d u+$ $C d x \wedge d z \wedge d u+D d y \wedge d z \wedge d u$ be any element of $\tilde{Z}^{3}=\Omega^{3}$. For this $\delta$, put $\rho=-\left(\int A d y\right) d x \wedge d z-\left(\int B d y\right) d x \wedge d u$ and put $\lambda=\left(C-\int B_{z} d y+\int A_{u} d y\right) d x \wedge$ $d z \wedge d u+D d y \wedge d z \wedge d u$. Then by Lemma 5.1(a), we have $\delta=d \rho+\lambda \in \tilde{B}^{3}+I^{3}$. This implies $H_{N P}^{3}\left(\eta_{1}\right)=0$.

Remark 5.1. In computing $H_{N P}^{1}\left(\eta_{1}\right)$ and $H_{N P}^{2}\left(\eta_{1}\right)$, we mentioned the last isomorphisms by using $\mathcal{I}_{\mathbb{R}^{2}}$ and $\mathcal{I}_{\mathbb{R}^{3}}$. The same facts also hold for $\eta_{2}, \eta_{3}$ and $\eta_{4}$.

For other Nambu-Poisson tensors $\eta_{i}, 2 \leq i \leq 7$, we can compute the corresponding Nambu-Poisson cohomologies by using the analogous methods as in the case of $\eta_{1}$ except for the slight modification. So we state only the results of computations by emphasizing the differences between the cases of $\eta_{i}, \quad 2 \leq i \leq 7$ and that of $\eta_{1}$.

The results including Theorem 5.5 are summarized in the following table. Each $H_{N P}^{*}$ is described under "isomorphism". For example, in $\eta_{1}$-case, we should read that $H_{N P}^{1}$ is "isomorphic" to $\tilde{\mathcal{F}} / \tilde{\mathcal{F}}_{1}$.

Table 3. Nambu-Poisson Cohomology of Type (II)

\begin{tabular}{c|c|c|c|c} 
cohomology & $H_{N P}^{0}$ & $H_{N P}^{1}$ & $H_{N P}^{2}$ & $H_{N P}^{k}, k \geq 3$ \\
\hline$\eta_{1}$ & $\mathbb{R}$ & $\tilde{\mathcal{F}} / \tilde{\mathcal{F}}_{1}$ & $\tilde{\mathcal{G}} / \tilde{\mathcal{G}}_{1}$ & 0 \\
\hline$\eta_{2}$ & $U \subset C^{\infty}(\mathbb{R})$ & $\tilde{\mathcal{F}} / \tilde{\mathcal{F}}_{2}$ & $\tilde{\mathcal{G}} / \tilde{\mathcal{G}}_{2}$ & 0 \\
\hline$\eta_{3}$ & $\mathbb{R}$ & $\tilde{\mathcal{F}} / \tilde{\mathcal{F}}_{3}$ & $\tilde{\mathcal{G}} / \tilde{\mathcal{G}}_{3}$ & 0 \\
\hline$\eta_{4}$ & $\mathbb{R}$ & $\tilde{\mathcal{F}} / \tilde{\mathcal{F}}_{4}$ & $\tilde{\mathcal{G}} / \tilde{\mathcal{G}}_{4}$ & 0 \\
\hline$\eta_{5}$ & $C^{\infty}\left(\mathbb{R}^{+}\right)$ & $C^{\infty}\left(\mathbb{R}^{+}\right)$ & $C^{\infty}\left(\mathbb{R}_{+}^{2}\right)$ & 0 \\
\hline$\eta_{6}$ & $C^{\infty}(\mathbb{R})$ & 0 & 0 & 0 \\
\hline$\eta_{7}$ & $C^{\infty}(\mathbb{R})$ & 0 & 0 & 0
\end{tabular}


In the above Table 3, we used the following notations:

- $U$ is a subspace of $C^{\infty}(\mathbb{R})$;

- $\tilde{\mathcal{F}}_{1}=\left\{(\alpha z+u) \tilde{h}_{z}+\alpha u \tilde{h}_{u}+2 \alpha \tilde{h} \mid \tilde{h} \in \tilde{\mathcal{F}}\right\}$;

- $\tilde{\mathcal{G}}_{1}=\left\{(\alpha z+u) \tilde{g}_{z}+\alpha u \tilde{g}_{u}+2 \alpha \tilde{g} \mid \tilde{g} \in \tilde{\mathcal{G}}\right\}$;

- $\tilde{\mathcal{F}}_{2}=\left\{(\alpha+\beta) \tilde{h}+\alpha z \tilde{h}_{z}+\beta u \tilde{h}_{u} \mid \tilde{h} \in \tilde{\mathcal{F}}\right\}$;

- $\tilde{\mathcal{G}}_{2}=\left\{\alpha z \tilde{g}_{z}+\beta u \tilde{g}_{u}+(\alpha+\beta) \tilde{g} \mid \tilde{g} \in \tilde{\mathcal{G}}\right\}$;

- $\tilde{\mathcal{F}}_{3}=\left\{(\alpha z-\beta u) \tilde{h}_{z}+(\beta z+\alpha u) \tilde{h}_{u}+2 \alpha \tilde{h} \mid \tilde{h} \in \tilde{\mathcal{F}}\right\}$;

- $\tilde{\mathcal{G}}_{3}=\left\{(\alpha z-\beta u) \tilde{g}_{z}+(\beta z+\alpha u) \tilde{g}_{u}+2 \alpha \tilde{g} \mid \tilde{g} \in \tilde{\mathcal{G}}\right\}$;

- $\tilde{\mathcal{F}}_{4}=\left\{z \tilde{h}_{z}+u \tilde{h}_{u}+2 \tilde{h} \mid \tilde{h} \in \tilde{\mathcal{F}}\right\}$

- $\tilde{\mathcal{G}}_{4}=\left\{z \tilde{g}_{z}+u \tilde{g}_{u}+2 \tilde{g} \mid \tilde{g} \in \tilde{\mathcal{G}}\right\}$;

- $C^{\infty}\left(\mathbb{R}^{+}\right)$is a subspace of $C^{\infty}(\mathbb{R})$ consisting of functions which are defined on $\mathbb{R}^{+}$;

- $C^{\infty}\left(\mathbb{R}_{+}^{2}\right)$ is a subspace of $C^{\infty}\left(\mathbb{R}^{2}\right)$ consisting of functions whose second variable is defined only on $\mathbb{R}^{+}$.

Remark 5.2. If we compute $H_{N P}^{*}$ in the category of formal functions (in short, in the formal category), we have the following results.

(1) In cases of $\eta_{1}, \eta_{3}$ and $\eta_{4}$, then we have $H_{N P}^{1}=H_{N P}^{2}=0$.

(2) In case of $\eta_{2}$, put $U=H_{N P}^{0}$. If $\alpha \beta>0$, then $U \cong \mathbb{R}$. On the contrary, if $\alpha$ and $\beta$ are integers which satisfy $\alpha \beta<0$, then $U \cong C^{\infty}(\mathbb{R})$. Let $\alpha=q / p$ and $\beta=s / r$ be two irreducible rational numbers with $\alpha \beta<0$, and put $d=$ L.C.M of $\{p, r\}$. Then $U$ is a subspace of $C^{\infty}(\mathbb{R})$ generated by $\phi(t)=t^{k d}, \quad k=0,1,2, \ldots$

If $\beta / \alpha$ is a negative rational number, then $H_{N P}^{1}$ and $H_{N P}^{2}$ are infinite dimensional in the formal category. Hence they are also infinite dimensional in the $C^{\infty}$-category. If $\beta / \alpha$ is a positive rational number or an irrational number, then $H_{N P}^{1}=H_{N P}^{2}=0$ in the formal category.

\section{§5.4. Computation of Nambu-Poisson cohomology of type (III)}

By an easy consideration, we know that $\sharp_{2}\left(\Omega^{2}\right)=\mathfrak{g}_{\phi}$ is spanned by $\left\langle\phi \frac{\partial}{\partial x}\right.$, $\left.\phi \frac{\partial}{\partial y}, \phi \frac{\partial}{\partial z}\right\rangle$ over $\mathcal{F}$. Moreover we know that each $I^{i}, 1 \leq i \leq 4$ coincides with (f) of Lemma 5.1. Hence each Nambu-Poisson cohomology of $H_{N P}^{k}\left(\eta_{\phi}\right)$ of Type (III) is completely isomorphic to that of $H_{N P}^{k}\left(\eta_{6}\right)$. Thus we have

Proposition 5.6. Let $\eta_{\phi}=\phi \frac{\partial}{\partial x} \wedge \frac{\partial}{\partial y} \wedge \frac{\partial}{\partial z}$, where $\phi$ is a linear function on $\mathbb{R}^{4}$. Then we have

$$
\begin{aligned}
& H_{N P}^{0}\left(\eta_{\phi}\right) \cong C^{\infty}(\mathbb{R}), \\
& H_{N P}^{k}\left(\eta_{\phi}\right)=0, \quad k \geq 1 .
\end{aligned}
$$




\section{$\S 5.5$. Computation of Nambu-Poisson cohomology of type (VI)}

We will only treat here the generic case. Namely we suppose that there exists non-zero constant $k$ such that $b_{3}=k a_{3}, b_{4}=k a_{4}$. Then we have $p=-k(q+1)=-k(-k r+2)$ and $q-1=-k r$. Now a Nambu-Poisson tensor $\eta_{\psi}$ can be written as

$$
\eta_{\psi}=\left\{(-k r+2) x+r y+a_{3} z+a_{4} u\right\}\left(k \frac{\partial}{\partial y} \wedge \frac{\partial}{\partial z} \wedge \frac{\partial}{\partial u}+\frac{\partial}{\partial x} \wedge \frac{\partial}{\partial z} \wedge \frac{\partial}{\partial u}\right) .
$$

Then the Lie algebra $\mathfrak{g}$ corresponding to $\eta_{\psi}$ is as follows.

$$
\begin{gathered}
\mathfrak{g}=\left\langle x \frac{\partial}{\partial x}+k x \frac{\partial}{\partial y}, y \frac{\partial}{\partial x}+k y \frac{\partial}{\partial y}, z \frac{\partial}{\partial x}+k z \frac{\partial}{\partial y}, u \frac{\partial}{\partial x}+k u \frac{\partial}{\partial y}\right. \\
\left.x \frac{\partial}{\partial z}, y \frac{\partial}{\partial z}, z \frac{\partial}{\partial z}, u \frac{\partial}{\partial z}, x \frac{\partial}{\partial u}, y \frac{\partial}{\partial u}, z \frac{\partial}{\partial u}, u \frac{\partial}{\partial u}\right\rangle
\end{gathered}
$$

Recall that $I^{k}$ is a subspace of $\Omega^{k}$ whose element $c \in I^{k}$ satisfies $c(\mathfrak{g}, \ldots, \mathfrak{g})$ $=0$.

Lemma 5.7. Let $A, B, C, D, E \in \mathcal{F}$. Then we have

$$
\begin{aligned}
I^{1}= & \{A d x+B d y \mid A+k B=0\}, \\
I^{2}= & \{A d x \wedge d y+B d x \wedge d z+C d x \wedge d u+D d y \wedge d z \\
& +E d y \wedge d u \mid B+k D=0, C+k E=0\}, \\
I^{3}= & \{A d x \wedge d y \wedge d z+B d x \wedge d y \wedge d u+C d x \wedge d z \wedge d u \\
& +D d y \wedge d z \wedge d u \mid C+k D=0\}, \\
I^{4}= & \Omega^{4} .
\end{aligned}
$$

Proof. Straightforward calculation.

\section{Theorem 5.8.}

$$
\begin{aligned}
& H_{N P}^{0}\left(\eta_{\psi}\right) \cong C^{\infty}(\mathbb{R}), \\
& H_{N P}^{1}\left(\eta_{\psi}\right) \cong C^{\infty}\left(\mathbb{R}^{2}\right) / C^{\infty}(\mathbb{R}), \\
& H_{N P}^{2}\left(\eta_{\psi}\right) \cong C^{\infty}\left(\mathbb{R}^{3}\right) / C^{\infty}\left(\mathbb{R}^{2}\right), \\
& H_{N P}^{3}\left(\eta_{\psi}\right) \cong \mathcal{F} / C^{\infty}\left(\mathbb{R}^{3}\right), \\
& H_{N P}^{k}\left(\eta_{\psi}\right)=0, \quad k \geq 4,
\end{aligned}
$$

where $\mathcal{F}=C^{\infty}\left(\mathbb{R}^{4}\right)$. 
Proof. To compute $H_{N P}^{*}\left(\eta_{\psi}\right)$, we will use Proposition 5.2 again. The space $H_{N P}^{0}\left(\eta_{\psi}\right)$ is consisting of functions $f \in \mathcal{F}$ which are $\mathfrak{g}$-invariant. Hence each $f \in H_{N P}^{0}\left(\eta_{\psi}\right)$ must satisfy $f=f(x, y)$ and $r \cdot f_{x}+k \cdot r \cdot f_{y}=0$ for any linear function $r$ on $\mathbb{R}^{4}$. These conditions are easily lead us to the fact that $f=\phi(k x-y)$, where $\phi$ is any $C^{\infty}$-function of one variable. Hence $H_{N P}^{0}\left(\eta_{\psi}\right) \cong$ $C^{\infty}(\mathbb{R})$.

Next let us compute $H_{N P}^{1}\left(\eta_{\psi}\right)$. Put $c=A d x+B d y+C d z+D d u$. Then $c \in \tilde{Z}^{1}$ if and only if

$$
\left\{\begin{array}{c}
D_{z}=C_{u}, \\
C_{x}-A_{z}+k\left(C_{y}-B_{z}\right)=0, \\
D_{x}-A_{u}+k\left(D_{y}-B_{u}\right)=0 .
\end{array}\right.
$$

By the first equation, there exists a function $h \in \mathcal{F}$ such that $C=h_{z}, \quad D=$ $h_{u}$. Substituting these equations into second and third equations, we have

$$
\begin{aligned}
& \frac{\partial}{\partial z}\left(h_{x}-A+k\left(h_{y}-B\right)\right)=0, \\
& \frac{\partial}{\partial u}\left(h_{x}-A+k\left(h_{y}-B\right)\right)=0 .
\end{aligned}
$$

Hence we know that there exists a function $S(x, y)$ such that

$$
A=h_{x}+k h_{y}-k B-S(x, y) .
$$

Then $c \in \tilde{Z}^{1}$ can be rewritten as follows.

$$
\begin{aligned}
c & =\left(h_{x}+k h_{y}-k B-S(x, y)\right) d x+B d y+h_{z} d z+h_{u} d u \\
& =d h+k h_{y} d x-h_{y} d y-k B d x+B d y-S(x, y) d x .
\end{aligned}
$$

Since $d h+k h_{y} d x-h_{y} d y-k B d x+B d y$ is an element of $\tilde{B}^{1}+I^{1}$ by Lemma 5.7 , we have $c \equiv-S(x, y) d x\left(\bmod \tilde{B}^{1}+I^{1}\right)$. Moreover $S(x, y) d x \in \tilde{B}^{1}$ if and only if $S(x, y)=S(x)$. Hence we finally obtain that $H_{N P}^{1}\left(\eta_{\psi}\right) \cong C^{\infty}\left(\mathbb{R}^{2}\right) / C^{\infty}(\mathbb{R})$ by Proposition 5.2.

Next let us compute $H_{N P}^{2}\left(\eta_{\psi}\right)$. By Proposition 5.2, $c=A d x \wedge d y+B d x \wedge$ $d z+C d x \wedge d u+D d y \wedge d z+E d y \wedge d u+F d z \wedge d u$ is contained in $\tilde{Z}^{2}$ if and only if

$$
B_{u}-C_{z}+F_{x}+k\left(D_{u}-E_{z}+F_{y}\right)=0 .
$$

This equation is equivalent to

$$
B+k D=\int(C+k E)_{z} d u-\int\left(F_{x}+k F_{y}\right) d u+\phi(x, y, z),
$$


for some $C^{\infty}$-function $\phi(x, y, z)$. Since $A d x \wedge d y-k D d x \wedge d z-k E d x \wedge d u+$ $D d y \wedge d z+E d y \wedge d u$ is an element of $I^{2}$ by Lemma 5.7, we have

$$
c \equiv(B+k D) d x \wedge d z+(C+k E) d x \wedge d u+F d z \wedge d u\left(\bmod I^{2}\right) .
$$

Thus $c \in \tilde{Z}^{2}$ can be rewritten as follows.

$$
\begin{aligned}
c \equiv & \left(\int(C+k E)_{z} d u-\int\left(F_{x}+k F_{y}\right) d u+\phi(x, y, z)\right) d x \wedge d z \\
& +(C+k E) d x \wedge d u+F d z \wedge d u\left(\bmod I^{2}\right) .
\end{aligned}
$$

Put $\rho=-\left(\int(C+k E) d u\right) d x$, and $\delta=-\left(\int F d u\right) d z$. Then

$$
\begin{aligned}
\tilde{B}^{2} \ni d \rho= & (C+k E) d x \wedge d u+\left(\int(C+k E)_{y} d u\right) d x \wedge d y \\
& +\left(\int(C+k E)_{z} d u\right) d x \wedge d z
\end{aligned}
$$

and

$$
\begin{aligned}
\tilde{B}^{2} \ni d \delta= & -\left(\int F_{x} d u\right) d x \wedge d z-\left(\int F_{y} d u\right) d y \wedge d z \\
& +F d z \wedge d u
\end{aligned}
$$

Hence

$$
\begin{aligned}
c \equiv & -\left(\int(C+k E)_{y} d u\right) d x \wedge d y-k\left(\int F_{y} d u\right) d x \wedge d z \\
& +\left(\int F_{y} d u\right) d y \wedge d z+\phi(x, y, z) d x \wedge d z\left(\bmod \tilde{B}^{2}+I^{2}\right) .
\end{aligned}
$$

Recall that $-k\left(\int F_{y} d u\right) d x \wedge d z+\left(\int F_{y} d u\right) d y \wedge d z$, and $-\left(\int(C+k E)_{y} d u\right) d x \wedge d y$ are elements of $I^{2}$. Thus $c \equiv \phi(x, y, z) d x \wedge d z\left(\bmod \tilde{B}^{2}+I^{2}\right) . \phi(x, y, z) d x \wedge d z$ is an exact 2 -form if and only if $\phi(x, y, z)=\phi(x, z)$. Thus we have $H_{N P}^{2}\left(\eta_{\psi}\right) \cong$ $C^{\infty}\left(\mathbb{R}^{3}\right) / C^{\infty}\left(\mathbb{R}^{2}\right)$.

To compute $H_{N P}^{3}\left(\eta_{\psi}\right)$, put $c=A d x \wedge d y \wedge d z+B d x \wedge d y \wedge d u+C d x \wedge$ $d z \wedge d u+D d y \wedge d z \wedge d u \in \tilde{Z}^{3}=\Omega^{3}$. Since $A d x \wedge d y \wedge d z+B d x \wedge d y \wedge d u-$ $k D d x \wedge d z \wedge d u+D d y \wedge d z \wedge d u$ is contained in $I^{3}$ by Lemma 5.7, we have $c \equiv(C+k D) d x \wedge d z \wedge d u\left(\bmod I^{3}\right)$. Note that 3 -form $(C+k D) d x \wedge d z \wedge d u$ is contained in $\tilde{B}^{3}$ if and only if $\frac{\partial}{\partial y}(C+k D)=0$. Then using Proposition 5.2, we have $H_{N P}^{3}\left(\eta_{\psi}\right) \cong \mathcal{F} / C^{\infty}\left(\mathbb{R}^{3}\right)$.

For $k \geq 4$, it is clear that $H_{N P}^{k}\left(\eta_{\psi}\right)=0$, since $\Lambda^{k} \mathfrak{g}=0$. 


\section{§6. Computation of Nambu-Poisson Cohomology: Quadratic Case}

\section{$\S 6.1$. Notation and general remarks}

In this section we compute Nambu-Poisson cohomology in the case of quadratic Nambu-Poisson tensor. Let us consider $\eta=\left(x^{2}+y^{2}+z^{2}+u^{2}\right) \frac{\partial}{\partial x} \wedge \frac{\partial}{\partial y} \wedge$ $\frac{\partial}{\partial z}$, which is a Nambu-Poisson tensor of order 3 on $\mathbb{R}^{4}(x, y, z, u)$. As usual, we denote the Nambu-Poisson cohomology of $\left(\mathbb{R}^{4}, \eta\right)$ by $H_{N P}^{*}\left(\mathbb{R}^{4}, \eta\right)$. To compute $H_{N P}^{*}\left(\mathbb{R}^{4}, \eta\right)$, we will essentially use the result of computations of $H_{N P}^{*}\left(\mathbb{R}^{3}, \eta^{\prime}\right)$, where $\eta^{\prime}=\left(x^{2}+y^{2}+z^{2}\right) \frac{\partial}{\partial x} \wedge \frac{\partial}{\partial y} \wedge \frac{\partial}{\partial z}$.

First of all we review an equivalent cohomology to Nambu-Poisson cohomology, which is due to P. Monnier [9]. Let $M$ be an $m$-dimensional $C^{\infty}$ manifold with a volume form $\Omega$. For $h \in C^{\infty}(M)$, we define the operator

$$
\begin{aligned}
d_{h}: \Omega^{k}(M) & \longrightarrow \Omega^{k+1}(M) \\
\alpha & \mapsto h d \alpha-k d h \wedge \alpha .
\end{aligned}
$$

It is easy to prove that $d_{h} \circ d_{h}=0$. We denote by $H_{h}^{*}(M)$ the cohomology of this complex. Let $\eta$ be an element of $\Gamma\left(\Lambda^{m}(T M)\right)$. Recall that such $\eta$ becomes always a Nambu-Poisson tensor [10]. Then P. Monnier proved the following [9].

Proposition 6.1. If we put $h=i_{\eta} \Omega$, then $H_{N P}^{*}(M, \eta)$ is isomorphic to $H_{h}^{*}(M)$.

Remark 6.1. It is easy to see that if $g$ is a function on $M$ which does not vanish on $M$, then the cohomologies $H_{h}^{*}(M)$ and $H_{h g}^{*}(M)$ are isomorphic.

Throughout this section, we will use the following notations:

- $\mathcal{F}$ is the algebra of real-valued $C^{\infty}$ functions on $\mathbb{R}^{4}(x, y, z, u)$;

- $\mathcal{F}^{\prime}$ is the algebra of real-valued $C^{\infty}$ functions on $\mathbb{R}^{3}(x, y, z)$;

- $\chi\left(\mathbb{R}^{4}\right)$ is the $\mathcal{F}$-module of vector fields on $\mathbb{R}^{4}$;

- $\chi^{\prime}\left(\mathbb{R}^{4}\right)=\left\{A \frac{\partial}{\partial x}+B \frac{\partial}{\partial y}+C \frac{\partial}{\partial z} \mid A, B, C \in \mathcal{F}\right\}$;

- $f=x^{2}+y^{2}+z^{2}+u^{2}$;

- $f^{\prime}=x^{2}+y^{2}+z^{2}$

- $\Omega^{k}=$ the space of $k$-forms on $\mathbb{R}^{4}$;

- $\Omega_{1}^{\prime}=\{A d x+B d y+C d z \mid A, B, C \in \mathcal{F}\}$

- $\Omega_{2}^{\prime}=\{A d y \wedge d z+B d z \wedge d x+C d x \wedge d y \mid A, B, C \in \mathcal{F}\}$;

- $\Omega_{3}^{\prime}=\{A d x \wedge d y \wedge d z \mid A \in \mathcal{F}\}$. 
If we choose $\Omega=d x \wedge d y \wedge d z$ as the volume form on $\mathbb{R}^{3}$, then we have $f^{\prime}=i_{\eta^{\prime}} \Omega$. First we compute $H_{N P}^{*}\left(\mathbb{R}^{3}, \eta^{\prime}\right)$, which is isomorphic to $H_{f^{\prime}}^{*}\left(\mathbb{R}^{3}\right)$ by Proposition 6.1. In the formal category (i.e. all coefficients of differential forms are formal power series), the following results were obtained by P. Monnier [9].

Proposition 6.2. In the formal case, $H_{f^{\prime}}^{0} \cong \mathbb{R}, H_{f^{\prime}}^{1} \cong \mathbb{R}, H_{f^{\prime}}^{2}=0$ and $H_{f^{\prime}}^{3} \cong \mathbb{R}$.

We want to compute $H_{f^{\prime}}^{*}$ in the $C^{\infty}$-category, and we will show that Proposition 6.2 still holds even in the $C^{\infty}$-category. First it is clear that $H_{f^{\prime}}^{0} \cong \mathbb{R}$. R. Ibáñez et al. [7] proved independently of P. Monnier [9] that $H_{f^{\prime}}^{1} \cong \mathbb{R}$. Hence it only remains to compute $H_{f^{\prime}}^{2}$ and $H_{f^{\prime}}^{3}$. To compute them, we use Proposition 6.2.

Let $\beta$ be a 2 -cocycle. Then by definition, $\beta$ satisfies $f^{\prime} d \beta=2 d f^{\prime} \wedge \beta$. Denote by $[\beta]$ the formal Taylor expansion of $\beta$ at the origin. Then by Proposition 6.2, there exists a formal 1 -form $[\alpha]$ such that $[\beta]=f^{\prime} d[\alpha]-d f^{\prime} \wedge[\alpha]$. Hence we can find a 1-form $\alpha$, whose formal Taylor expansion at the origin is $[\alpha]$. Put $\beta^{\prime}=\beta-\left(f^{\prime} d \alpha-d f^{\prime} \wedge \alpha\right)$. Then $\beta^{\prime}$ is flat (i.e. $\left.\left[\beta^{\prime}\right]=0\right)$ and satisfies $f^{\prime} d \beta^{\prime}=2 d f^{\prime} \wedge \beta^{\prime}$. $\frac{\beta^{\prime}}{f^{\prime 2}}$ is also flat and $d\left(\frac{\beta^{\prime}}{f^{\prime 2}}\right)=\frac{1}{f^{\prime 3}}\left(f^{\prime} d \beta^{\prime}-2 d f^{\prime} \wedge \beta^{\prime}\right)=0$. Hence there exists a flat 1 -form $\tilde{\alpha}$ such that $\frac{\beta^{\prime}}{f^{\prime 2}}=d \tilde{\alpha}$. Put $\tilde{\alpha}=\frac{\alpha^{\prime}}{f^{\prime}}$. Then $\alpha^{\prime}$ is a flat 1-form, and we get $\beta^{\prime}=f^{\prime 2} d \tilde{\alpha}=f^{\prime} d \alpha^{\prime}-d f^{\prime} \wedge \alpha^{\prime}$. Finally we have

$$
\beta=f^{\prime} d\left(\alpha+\alpha^{\prime}\right)-d f^{\prime} \wedge\left(\alpha+\alpha^{\prime}\right)
$$

This means $H_{f^{\prime}}^{2}=0$.

Next let us compute $H_{f^{\prime}}^{3}$. The space of 3-cocycles $Z_{f^{\prime}}^{3}$ is clearly isomorphic to $\mathcal{F}^{\prime}$. And the space of 3 -coboundaries $B_{f^{\prime}}^{3}$ is isomorphic to the following space $\mathcal{F}_{1}$.

$$
\mathcal{F}_{1}=\left\{f^{\prime}\left(\frac{\partial A}{\partial x}+\frac{\partial B}{\partial y}+\frac{\partial C}{\partial z}\right)-4(x A+y B+z C) ; A, B, C \in \mathcal{F}^{\prime}\right\} .
$$

Lemma 6.3. $\quad$ Let $\mathcal{I}$ be the subspace of $\mathcal{F}^{\prime}$ consisting of functions which are flat at the origin. Then $\mathcal{I} \subset \mathcal{F}_{1}$.

Proof. For $q \in \mathcal{I}$, put

$$
A=\left(f^{\prime}\right)^{2} \int \frac{q}{\left(f^{\prime}\right)^{3}} d x, B=0, C=0 .
$$

Then $f^{\prime}\left(\frac{\partial A}{\partial x}+\frac{\partial B}{\partial y}+\frac{\partial C}{\partial z}\right)-4(x A+y B+z C)=q$. Hence we have that $q \in \mathcal{F}_{1}$. 
Denote by $F^{\prime}$ (resp. $F_{1}$ ) the formal algebra corresponding to $\mathcal{F}^{\prime}$ (resp. $\left.\mathcal{F}_{1}\right)$. Let $T$ be a mapping from $\mathcal{F}^{\prime}$ to $F^{\prime}$, where $T(h)$ is the formal Taylor expansion of $h$ at the origin. Let $\pi: F^{\prime} \rightarrow F^{\prime} / F_{1}$ be the canonical projection, and put $\tilde{T}=\pi \circ T$. Then $\tilde{T}$ is a surjective linear mapping and it is clear that $\operatorname{ker} \tilde{T}=\mathcal{F}_{1}$ by Lemma 6.3 . Since $F^{\prime} / F_{1} \cong \mathbb{R}$ by Proposition 6.2 , we get that

$$
H_{f^{\prime}}^{3} \cong \mathcal{F}^{\prime} / \mathcal{F}_{1} \cong F^{\prime} / F_{1} \cong \mathbb{R}
$$

Thus we obtained the following proposition.

Proposition 6.4. In $C^{\infty}$-case, it still holds that $H_{f^{\prime}}^{0} \cong \mathbb{R}, H_{f^{\prime}}^{1} \cong \mathbb{R}$, $H_{f^{\prime}}^{2}=0$ and $H_{f^{\prime}}^{3} \cong \mathbb{R}$. that

For the Nambu-Poisson tensor $\eta=f \frac{\partial}{\partial x} \wedge \frac{\partial}{\partial y} \wedge \frac{\partial}{\partial z}$ defined on $\mathbb{R}^{4}$, we know

$$
\sharp_{2}\left(\Omega^{2}\right)=\left\{f X \mid X \in \chi^{\prime}\left(\mathbb{R}^{4}\right)\right\} .
$$

$\sharp_{2}\left(\Omega^{2}\right)$ is denoted by $\mathfrak{g}$, which is isomorphic to $\Omega^{2} /$ ker $\sharp_{2}$. Note also that $\Omega^{2} /$ ker $\sharp_{2}$ is isomorphic to $\Omega_{2}^{\prime}$. $\mathfrak{g}$ is, of course, a Lie subalgebra of $\chi\left(\mathbb{R}^{4}\right)$.

Since $H_{N P}^{0}\left(\mathbb{R}^{4}, \eta\right)=\{g \in \mathcal{F} \mid X g=0$ for all $X \in \mathfrak{g}\}$, it is clear that $H_{N P}^{0}\left(\mathbb{R}^{4}, \eta\right) \cong C^{\infty}(\mathbb{R})$.

In computing Nambu-Poisson cohomology, we use Proposition 6.4. To do this, we need the formal Taylor expansion of a function $A \in \mathcal{F}$ with respect to the variable $u$, which is denoted by $\bar{A}$. In other words, three variables $x, y$ and $z$ are regarded as parameters. And we say that $\bar{A}$ is the $u$-formal Taylor expansion of $A$. This terminology will be also used for differential forms and vector fields. Thus we can express $\bar{A}$ (similarly $\bar{B}$ and $\bar{C}$ ) as follows.

$$
\left\{\begin{array}{c}
\bar{A}=a_{0}+u a_{1}+u^{2} a_{2}+\cdots, \\
\bar{B}=b_{0}+u b_{1}+u^{2} b_{2}+\cdots, \\
\bar{C}=c_{0}+u c_{1}+u^{2} c_{2}+\cdots,
\end{array}\right.
$$

where $a_{k}, b_{k}, c_{k} \in \mathcal{F}^{\prime}$.

To compute $H_{N P}^{k}\left(\mathbb{R}^{4}, \eta\right), \quad k \geq 1$, let us define a linear mapping $d^{\prime}: \mathcal{F} \rightarrow \Omega_{1}^{\prime}$ by

$$
d^{\prime} g=\frac{\partial g}{\partial x} d x+\frac{\partial g}{\partial y} d y+\frac{\partial g}{\partial z} d z
$$

This operator $d^{\prime}$ is naturally extended to a linear mapping from $\Omega_{k}^{\prime}$ to $\Omega_{k+1}^{\prime}$. Moreover we define $d_{f}^{\prime}: \Omega_{k}^{\prime} \rightarrow \Omega_{k+1}^{\prime}$ by

$$
d_{f}^{\prime}(\alpha)=f d^{\prime} \alpha-k d^{\prime} f \wedge \alpha, \alpha \in \Omega_{k}^{\prime} .
$$


Then $d_{f}^{\prime} \circ d_{f}^{\prime}=0$, and we denote by $H_{d_{f}^{\prime}}^{*}$ the cohomology space with respect to $d_{f}^{\prime}$.

If we define $b: \chi^{\prime}\left(\mathbb{R}^{4}\right) \rightarrow \Omega_{2}^{\prime}$ by $b(X)=i(X) d x \wedge d y \wedge d z$, then we obtain that $\sharp_{2}(b(X))=f X$ and that $\sharp_{2}(\{b(X), b(Y)\})=\left[\sharp_{2}(b(X)), \sharp_{2}(b(Y))\right]=$ $[f X, f Y]$.

Following the similar method of P. Monnier [9], if $\phi: C^{k}\left(\Omega_{2}^{\prime}, \mathcal{F}\right) \rightarrow \Omega_{k}^{\prime}$ is defined by

$$
\phi\left(c^{k}\right)\left(X_{1}, \ldots, X_{k}\right)=c^{k}\left(b\left(X_{1}\right), \ldots, b\left(X_{k}\right)\right), X_{1}, \ldots, X_{k} \in \chi^{\prime}\left(\mathbb{R}^{4}\right),
$$

then $\phi$ is a linear isomorphism and we can prove the following.

Proposition 6.5. The following diagram is commutative.

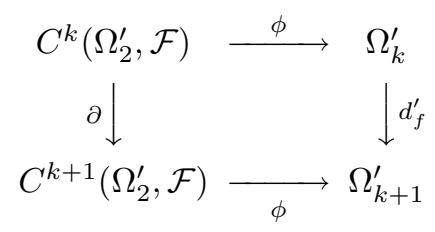

Hence $H_{N P}^{*}\left(\mathbb{R}^{4}, \eta\right) \cong H_{d_{f}^{\prime}}^{*}$.

Proof. We prove only for the case $k=1$. For $c \in C^{1}\left(\Omega_{2}^{\prime}, \mathcal{F}\right)$, put $\phi(c)=\alpha$. For any $X, Y \in \chi^{\prime}\left(\mathbb{R}^{4}\right)$, we can directly get

$$
\{b(X), b(Y)\}=f \cdot b([X, Y])-(X f) \cdot b(Y)+(Y f) \cdot b(X),
$$

from the definition of the bracket $\{$,$\} on \Omega_{2}^{\prime}$. Using this equation, we have

$$
\begin{aligned}
\phi(\partial c)(X, Y) & =(\partial c)(b(X), b(Y)) \\
& =f X \cdot c(b(Y))-f Y \cdot c(b(X))-c(\{b(X), b(Y)\}) \\
& =f X \cdot \alpha(Y)-f Y \cdot \alpha(X)-c(f \cdot b([X, Y]) \\
& +(X f) \cdot b(Y)-(Y f) \cdot b(X)) \\
& =f X \cdot \alpha(Y)-f Y \cdot \alpha(X)-f \alpha([X, Y]) \\
& -(X f) \cdot \alpha(Y)+(Y f) \cdot \alpha(X) \\
& =f \cdot d^{\prime} \alpha(X, Y)-\left(d^{\prime} f \wedge \alpha\right)(X, Y) \\
& =\left(d_{f}^{\prime} \alpha\right)(X, Y)=\left(d_{f}^{\prime} \circ \phi(c)\right)(X, Y) .
\end{aligned}
$$

Thus $\phi \circ \partial=d_{f}^{\prime} \circ \phi$. 


\section{$\S 6.2$. Computation of $H_{N P}^{1}\left(\mathbb{R}^{4}, \eta\right)$}

In this subsection, we compute $H_{N P}^{1}\left(\mathbb{R}^{4}, \eta\right)$. In order to do this, we have only to compute $H_{d_{f}^{\prime}}^{1}$ by Proposition 6.5. The space of 1-coboundaries, which is denoted by $B_{1}^{\prime}$, is the set of 1 -forms $f d^{\prime} g, g \in \mathcal{F}$. Let $Z_{1}^{\prime}$ be the space of 1-cocycles. Then for $\alpha=A d x+B d y+C d z \in \Omega_{1}^{\prime}, \alpha$ is an element of $Z_{1}^{\prime}$ if and only if $f d^{\prime} \alpha=d^{\prime} f \wedge \alpha$. This equation is equivalent to the following three equations.

$$
\left\{\begin{array}{l}
f \cdot\left(\frac{\partial B}{\partial x}-\frac{\partial A}{\partial y}\right)=2 x B-2 y A \\
f \cdot\left(\frac{\partial C}{\partial y}-\frac{\partial B}{\partial z}\right)=2 y C-2 z B \\
f \cdot\left(\frac{\partial A}{\partial z}-\frac{\partial C}{\partial x}\right)=2 z A-2 x C
\end{array}\right.
$$

Note that the $u$-formal Taylor expansion of $\alpha$ is written as $\bar{\alpha}=\alpha_{0}+u \alpha_{1}+$ $u^{2} \alpha_{2}+\cdots$, where $\alpha_{p}=a_{p} d x+b_{p} d y+c_{p} d z, a_{p}, b_{p}, c_{p} \in \mathcal{F}^{\prime}$. And three equations (4) induce the $u$-formal Taylor expansions. Comparing constant terms with respect to $u$ in them, we have

$$
\left\{\begin{array}{l}
f^{\prime} \cdot\left(\frac{\partial b_{0}}{\partial x}-\frac{\partial a_{0}}{\partial y}\right)=2 x b_{0}-2 y a_{0} \\
f^{\prime} \cdot\left(\frac{\partial c_{0}}{\partial y}-\frac{\partial b_{0}}{\partial z}\right)=2 y c_{0}-2 z b_{0} \\
f^{\prime} \cdot\left(\frac{\partial a_{0}}{\partial z}-\frac{\partial c_{0}}{\partial x}\right)=2 z a_{0}-2 x c_{0}
\end{array}\right.
$$

These three equations (5) essentially appeared in computing $H_{N P}^{1}\left(\mathbb{R}^{3}, \eta^{\prime}=\right.$ $\left.f^{\prime} \frac{\partial}{\partial x} \wedge \frac{\partial}{\partial y} \wedge \frac{\partial}{\partial z}\right)$. By Proposition 6.4, $H_{N P}^{1}\left(\mathbb{R}^{3}, \eta^{\prime}\right)$ is isomorphic to $\mathbb{R}$. The generator of $H_{N P}^{1}\left(\mathbb{R}^{3}, \eta^{\prime}\right)$ is $d f^{\prime}$ and this means that there exist a real number $k_{0}$ and a function $g_{0} \in \mathcal{F}^{\prime}$ such that

$$
\left\{\begin{array}{l}
a_{0}=k_{0} \cdot 2 x+f^{\prime} \cdot \frac{\partial g_{0}}{\partial x} \\
b_{0}=k_{0} \cdot 2 y+f^{\prime} \cdot \frac{\partial g_{0}}{\partial y} \\
c_{0}=k_{0} \cdot 2 z+f^{\prime} \cdot \frac{\partial g_{0}}{\partial z} .
\end{array}\right.
$$

Since $\alpha_{0}=a_{0} d x+b_{0} d y+c_{0} d z$, we obtain that $\alpha_{0}=k_{0} d f^{\prime}+f^{\prime} d g_{0}$. Similarly if we compare the coefficients of $u$ in the $u$-formal Taylor expansions, we can 
get $\alpha_{1}=k_{1} d f^{\prime}+f^{\prime} d g_{1}$, where $k_{1} \in \mathbb{R}$ and $g_{1} \in \mathcal{F}^{\prime}$. But if we compare the coefficients of $u^{2}$, the situation is slightly different. In fact, we have

$$
\left\{\begin{array}{l}
f^{\prime} \cdot\left(\frac{\partial b_{2}}{\partial x}-\frac{\partial a_{2}}{\partial y}\right)+\left(\frac{\partial b_{0}}{\partial x}-\frac{\partial a_{0}}{\partial y}\right)=2 x b_{2}-2 y a_{2} \\
f^{\prime} \cdot\left(\frac{\partial c_{2}}{\partial y}-\frac{\partial b_{2}}{\partial z}\right)+\left(\frac{\partial c_{0}}{\partial y}-\frac{\partial b_{0}}{\partial z}\right)=2 y c_{2}-2 z b_{2} \\
f^{\prime} \cdot\left(\frac{\partial a_{2}}{\partial z}-\frac{\partial c_{2}}{\partial x}\right)+\left(\frac{\partial a_{0}}{\partial z}-\frac{\partial c_{0}}{\partial x}\right)=2 z a_{2}-2 x c_{2}
\end{array}\right.
$$

These equations (7) can be rewritten as follows.

$$
\left\{\begin{array}{l}
f^{\prime}\left(\frac{\partial\left(b_{2}-\frac{\partial g_{0}}{\partial y}\right)}{\partial x}-\frac{\partial\left(a_{2}-\frac{\partial g_{0}}{\partial x}\right)}{\partial y}\right)=2 x\left(b_{2}-\frac{\partial g_{0}}{\partial y}\right)-2 y\left(a_{2}-\frac{\partial g_{0}}{\partial x}\right) \\
f^{\prime}\left(\frac{\partial\left(c_{2}-\frac{\partial g_{0}}{\partial z}\right)}{\partial y}-\frac{\partial\left(b_{2}-\frac{\partial g_{0}}{\partial y}\right)}{\partial z}\right)=2 y\left(c_{2}-\frac{\partial g_{0}}{\partial z}\right)-2 z\left(b_{2}-\frac{\partial g_{0}}{\partial y}\right) \\
f^{\prime}\left(\frac{\partial\left(a_{2}-\frac{\partial g_{0}}{\partial x}\right)}{\partial z}-\frac{\partial\left(c_{2}-\frac{\partial g_{0}}{\partial z}\right)}{\partial x}\right)=2 z\left(a_{2}-\frac{\partial g_{0}}{\partial x}\right)-2 x\left(c_{2}-\frac{\partial g_{0}}{\partial z}\right)
\end{array}\right.
$$

Thus we can apply Proposition 6.4 to (8), and we have that there exist a real number $k_{2}$ and $g_{2} \in \mathcal{F}^{\prime}$ such that

$$
\left\{\begin{array}{c}
a_{2}-\frac{\partial g_{0}}{\partial x}=k_{2} \cdot 2 x+f^{\prime} \frac{\partial g_{2}}{\partial x} \\
b_{2}-\frac{\partial g_{0}}{\partial y}=k_{2} \cdot 2 y+f^{\prime} \frac{\partial g_{2}}{\partial y} \\
c_{2}-\frac{\partial g_{0}}{\partial z}=k_{2} \cdot 2 z+f^{\prime} \frac{\partial g_{2}}{\partial z}
\end{array}\right.
$$

Hence $\alpha_{2}=k_{2} d f^{\prime}+f^{\prime} d g_{2}+d g_{0}$. By the same methods, we know that each $\alpha_{p}, \quad(p \geq 3)$ has the form $\alpha_{p}=k_{p} d f^{\prime}+f^{\prime} d g_{p}+d g_{p-2}$, where $k_{p} \in \mathbb{R}$ and $g_{p-2}, g_{p} \in \mathcal{F}^{\prime}$. These mean that $\bar{\alpha}$ has the following expression. Note that $d f^{\prime}=d^{\prime} f$ and that $f^{\prime}+u^{2}=f$.

$$
\bar{\alpha}=\left(k_{0}+k_{1} u+k_{2} u^{2}+\cdots\right) d^{\prime} f+f \cdot d^{\prime}\left(g_{0}+u g_{1}+u^{2} g_{2}+\cdots\right) .
$$

To obtain the final result, we need the following lemma, which is a generalization of E. Borel theorem. This will be proved in the analogous way as K. Abe and K. Fukui, Lemma 4.4 [1]. (See also R. Narasimhan [12], §1.5.2 and $\S 1$.5.3.) We put $\vec{r}=(x, y, z, u)$ and $|\vec{r}|=\sqrt{x^{2}+y^{2}+z^{2}+u^{2}}$. Then a function $F(\vec{r}) \in C^{\infty}\left(\mathbb{R}^{4}\right)$ is said to be $m$-flat as a function of $u$ at $(x, y, z, 0)$ if $\frac{\partial^{\alpha}}{\partial u^{\alpha}} F(x, y, z, 0)=0$ for $\alpha \leq m$. 
Lemma 6.6. For each integer $p \geq 0$, let $c_{p}(x, y, z) \in C^{\infty}\left(\mathbb{R}^{3}\right)$. Then there exists $G(\vec{r}) \in C^{\infty}\left(\mathbb{R}^{4}\right)$ such that the partial derivatives with respect to the last variable of $G$ at any point $(x, y, z, 0) \in \mathbb{R}^{4}$ are

$$
\frac{\partial^{p} G}{\partial u^{p}}(x, y, z, 0)=p ! c_{p}(x, y, z) \quad p \geq 0 .
$$

Proof. Let $T_{m}(\vec{r})=\sum_{p=0}^{m} c_{p}(x, y, z) u^{p}$ for $\vec{r} \in \mathbb{R}^{4}$. Let $H(\vec{r}) \in C^{\infty}\left(\mathbb{R}^{4}\right)$ such that $H(\vec{r})=0$ for $|\vec{r}| \leq 1 / 2, \quad H(\vec{r})=1$ for $|\vec{r}| \geq 1$ and $H(\vec{r}) \geq 0$ for any $\vec{r} \in \mathbb{R}^{4}$. For a positive number $\delta$, put

$$
g_{\delta}(\vec{r})=H\left(\frac{\vec{r}}{\delta}\right)\left(T_{m+1}(\vec{r})-T_{m}(\vec{r})\right) .
$$

Clearly $g_{\delta} \in C^{\infty}\left(\mathbb{R}^{4}\right)$ and vanishes near 0 . Moreover $T_{m+1}-T_{m}$ is $m$-flat as a function of $u$ at any point $(x, y, z, 0)$. Hence as in the proof of Lemma 1.5.2 [12], there exists a positive number $\delta_{m}$ such that

$$
\sum_{p=0}^{m} \frac{1}{p !}\left|\frac{\partial^{p}}{\partial u^{p}}\left(g_{\delta_{m}}-\left(T_{m+1}-T_{m}\right)\right)(\vec{r})\right|<2^{-m} .
$$

Put $g_{m}=g_{\delta_{m}}$. If we define

$$
G=T_{0}+\sum_{m=0}^{\infty}\left(T_{m+1}-T_{m}-g_{m}\right),
$$

then as in the proof of Lemma 1.5.3 [12], we get that the function $G$ is the desired function.

By Lemma 6.6, we obtain that there exist a $C^{\infty}$-function $k(u)$ and a $C^{\infty}$ function $g(x, y, z, u)$ such that $\overline{k(u)}=k_{0}+k_{1} u+k_{2} u^{2}+\cdots$, and $\overline{g(x, y, z, u)}=$ $g_{0}+u g_{1}+u^{2} g_{2}+\cdots$. Put $\alpha^{\prime}=k(u) d^{\prime} f+f d^{\prime} g$, and put $\alpha-\alpha^{\prime}=\alpha_{f}$. Then $\alpha_{f}$ is a 1-cocycle and it satisfies $\overline{\alpha_{f}}=0$ (u-flat 1 -form). Let $k_{1}(u)$ be a flat function of one variable $u$. Then $\left(\alpha_{f}-k_{1}(u) d^{\prime} f\right) / f$ is a well-defined 1 -form on $\mathbb{R}^{4}$, and it satisfies

$$
d^{\prime}\left(\frac{\alpha_{f}-k_{1}(u) d^{\prime} f}{f}\right)=\frac{1}{f^{2}}\left(f d^{\prime} \alpha_{f}-d^{\prime} f \wedge\left(\alpha_{f}-k_{1}(u) d^{\prime} f\right)\right)=0 .
$$

Hence, as is easily seen, there exists a flat function $\tilde{g}(x, y, z, u)$ such that $\left(\alpha_{f}-\right.$ $\left.k_{1}(u) d^{\prime} f\right) / f=d^{\prime} \tilde{g}$. And we obtain that $\alpha \in Z_{1}^{\prime}$ has the following form:

$$
\alpha=\alpha_{f}+\alpha^{\prime}=\left(k(u)+k_{1}(u)\right) d^{\prime} f+f d^{\prime}(g+\tilde{g}) .
$$


$\alpha$ is, by definition, cohomologous to $\left(k(u)+k_{1}(u)\right) d^{\prime} f$. Moreover $l(u) d^{\prime} f$ is contained in $B_{1}^{\prime}$ if and only if $l(u)$ is a flat function at $u=0$. In fact, note that in this case $l(u) \log f$ is a $C^{\infty}$-function and it holds that $l(u) d^{\prime} f=f d^{\prime}(l(u) \log f) \in$ $B_{1}^{\prime}$. Thus we obtain that $H_{N P}^{1}\left(\mathbb{R}^{4}, \eta\right)$ is isomorphic to $\mathbb{R}[[u]]$, which is the space of formal power series of one variable $u$.

\section{$\S 6.3$. Computation of $H_{N P}^{2}\left(\mathbb{R}^{4}, \eta\right)$}

We will compute $H_{N P}^{2}\left(\mathbb{R}^{4}, \eta\right)$. By Proposition 6.5 , we will compute $H_{d_{f}^{\prime}}^{2}$. Every computation proceeds in the analogous way as the case of $H_{d_{f}^{\prime}}^{1}$. The space of 2-coboundaries $B_{2}^{\prime}$ is, by definition, the set of 2-forms $d_{f}^{\prime} \gamma=f d^{\prime} \gamma-$ $d^{\prime} f \wedge \gamma, \gamma \in \Omega_{1}^{\prime}$. Let $Z_{2}^{\prime}$ be the space of 2-cocycles. Then for $\beta=A d y \wedge d z+$ $B d z \wedge d x+C d x \wedge d y \in \Omega_{2}^{\prime}, \beta$ is an element of $Z_{2}^{\prime}$ if and only if $f d^{\prime} \beta=2 d^{\prime} f \wedge \beta$. This is equivalent to

$$
f \cdot\left(\frac{\partial A}{\partial x}+\frac{\partial B}{\partial y}+\frac{\partial C}{\partial z}\right)=4(x A+y B+z C) .
$$

The $u$-formal Taylor expansion (with respect to $u$ ) of $\beta$ is written as $\bar{\beta}=$ $\beta_{0}+u \beta_{1}+u^{2} \beta_{2}+\cdots$, where $\beta_{p}=a_{p} d y \wedge d z+b_{p} d z \wedge d x+c_{p} d x \wedge d y, a_{p}, b_{p}, c_{p} \in \mathcal{F}^{\prime}$. Then the equation (10) has the $u$-formal Taylor expansion.

Comparing constant terms in it, we have

$$
f^{\prime} \cdot\left(\frac{\partial a_{0}}{\partial x}+\frac{\partial b_{0}}{\partial y}+\frac{\partial c_{0}}{\partial z}\right)=4\left(x a_{0}+y b_{0}+z c_{0}\right) .
$$

This is equivalent to $d_{f^{\prime}} \beta_{0}=0$ for $\beta_{0}=a_{0} d y \wedge d z+b_{0} d z \wedge d x+c_{0} d x \wedge d y$. Recall that $H_{N P}^{2}\left(\mathbb{R}^{3}, \eta^{\prime}\right)=0$ by Proposition 6.4. In other words, if $d_{f^{\prime}} \beta_{0}=0$, then $\beta_{0}$ must be a coboundary. This means that we can find a 1-form $\alpha_{0}$ such that $\beta_{0}=f^{\prime} d \alpha_{0}-d f^{\prime} \wedge \alpha_{0}$.

Comparing the coefficients of $u$, we can also find a 1-form $\alpha_{1}$ such that $\beta_{1}=f^{\prime} d \alpha_{1}-d f^{\prime} \wedge \alpha_{1}$. Moreover if $p \geq 2$ we can find $p$-form $\alpha_{p}$ such that $\beta_{p}=f^{\prime} d \alpha_{p}-d^{\prime} f \wedge \alpha_{p}+d \alpha_{p-2}$. The $u$-formal Taylor expansion of $\beta$ is as follows.

$$
\begin{aligned}
\bar{\beta} & =\sum_{p=0}^{\infty} u^{p} \beta_{p} \\
& =\sum_{p=0}^{\infty} u^{p}\left(f^{\prime} d \alpha_{p}-d^{\prime} f \wedge \alpha_{p}\right)+\sum_{p=0}^{\infty} u^{p+2} d \alpha_{p}
\end{aligned}
$$




$$
\begin{aligned}
& =\sum_{p=0}^{\infty} u^{p}\left(f^{\prime} d \alpha_{p}-d f^{\prime} \wedge \alpha_{p}+u^{2} d \alpha_{p}\right) \\
& =\sum_{p=0}^{\infty} u^{p}\left(f d \alpha_{p}-d^{\prime} f \wedge \alpha_{p}\right) \\
& =f d^{\prime}\left(\sum_{p=0}^{\infty} u^{p} \alpha_{p}\right)-d^{\prime} f \wedge\left(\sum_{p=0}^{\infty} u^{p} \alpha_{p}\right) .
\end{aligned}
$$

Put $\hat{\alpha}=\sum_{p=0}^{\infty} u^{p} \alpha_{p}$. Then $\bar{\beta}=f d^{\prime} \hat{\alpha}-d^{\prime} f \wedge \hat{\alpha}$. By Lemma 6.6, there exists a 1 -form $\alpha^{\prime} \in \Omega_{1}^{\prime}$ such that $\bar{\alpha}^{\prime}=\hat{\alpha}$. Put $\beta^{\prime}=f d^{\prime} \alpha^{\prime}-d^{\prime} f \wedge \alpha^{\prime}$. Then $\bar{\beta}=\bar{\beta}^{\prime}$ and hence if we put $\tilde{\beta}=\beta-\beta^{\prime}$, then $\tilde{\beta}$ is a flat 2 -form of $\Omega_{2}^{\prime}$. Moreover it is easy to see that $f d^{\prime} \tilde{\beta}=2 d^{\prime} f \wedge \tilde{\beta}$, which means $\tilde{\beta} \in Z_{2}^{\prime}$. Then by the same method as the proof of $H_{f^{\prime}}^{2}=0$ ( $C^{\infty}$-case $)$, we can prove that there exists a flat 1-form $\alpha_{2}$ such that $\tilde{\beta}=f d^{\prime} \alpha_{2}-d^{\prime} f \wedge \alpha_{2}$. Hence $\beta$ has the following form:

$$
\beta=\beta^{\prime}+\tilde{\beta}=f d^{\prime}\left(\alpha^{\prime}+\alpha_{2}\right)-d^{\prime} f \wedge\left(\alpha^{\prime}+\alpha_{2}\right),
$$

and thus $\beta \in B_{2}^{\prime}$. Hence we get $H_{N P}^{2}\left(\mathbb{R}^{4}, \eta\right)=0$.

\section{§6.4. Computation of $H_{N P}^{3}\left(\mathbb{R}^{4}, \eta\right)$}

Let $Z_{3}^{\prime}$ be the space of 3 -cocycles. Since $\Omega_{4}^{\prime}=0$, it holds that $Z_{3}^{\prime}=\Omega_{3}^{\prime}$. Hence $Z_{3}^{\prime}$ is isomorphic to $\mathcal{F}$. Let $B_{3}^{\prime}$ be the space of 3 -coboundaries. Then every element of $B_{3}^{\prime}$ is written as

$$
\begin{aligned}
d_{f}^{\prime} \beta & =f d^{\prime} \beta-2 d^{\prime} f \wedge \beta \\
& =\left\{f\left(\frac{\partial A}{\partial x}+\frac{\partial B}{\partial y}+\frac{\partial C}{\partial z}\right)-4(x A+y B+z C)\right\} d x \wedge d y \wedge d z,
\end{aligned}
$$

where $\beta=A d y \wedge d z+B d z \wedge d x+C d x \wedge d y$ is an arbitrary element of $\Omega_{2}^{\prime}$.

Put $\mathcal{B}=\left\{f\left(\frac{\partial A}{\partial x}+\frac{\partial B}{\partial y}+\frac{\partial C}{\partial z}\right)-4(x A+y B+z C) \mid A, B, C \in \mathcal{F}\right\}$. Then, by Proposition $6.5, H_{N P}^{3}\left(\mathbb{R}^{4}, \eta\right)$ is isomorphic to $\mathcal{F} / \mathcal{B}$.

Lemma 6.7. Put $\mathcal{I}=\left\{h \in \mathcal{F} \mid \frac{\partial^{p} h}{\partial u^{p}}(x, y, z, 0)=0, p \geq 0\right\}$. i.e., each element $h$ of $\mathcal{I}$ is $u$-flat. Then $\mathcal{I} \subset \mathcal{B}$.

Proof. For $h \in \mathcal{I}$, it is clear that $h / f^{3}$ is an element of $\mathcal{F}$. Put $A=$ $f^{2} \int \frac{h}{f^{3}} d x, B=0$ and $C=0$. Then we have

$$
f\left(\frac{\partial A}{\partial x}+\frac{\partial B}{\partial y}+\frac{\partial C}{\partial z}\right)-4(x A+y B+z C)=h .
$$

Hence $h \in \mathcal{B}$. 
Put $\hat{F}=\{\bar{A} \mid A \in \mathcal{F}\}$ and $\hat{B}=\{\bar{A} \mid A \in \mathcal{B}\}$. We also denote by $\mathcal{F}_{0}^{\prime}$ the subspace of functions $g(x, y, z) \in \mathcal{F}^{\prime}$ with $g(0,0,0)=0$.

Proposition 6.8. $\quad \hat{F} / \hat{B} \cong \mathbb{R}[[u]]$.

Proof. For any element $g=f\left(\frac{\partial A}{\partial x}+\frac{\partial B}{\partial y}+\frac{\partial C}{\partial z}\right)-4(x A+y B+z C) \in \mathcal{B}$, its $u$-formal Taylor expansion is

$$
\begin{aligned}
\hat{B} \ni \bar{g} & =f\left(\frac{\partial \bar{A}}{\partial x}+\frac{\partial \bar{B}}{\partial y}+\frac{\partial \bar{C}}{\partial z}\right)-4(x \bar{A}+y \bar{B}+z \bar{C}) \\
& =\sum_{p=0}^{\infty}\left[u^{p}\left\{f^{\prime}\left(\frac{\partial a_{p}}{\partial x}+\frac{\partial b_{p}}{\partial y}+\frac{\partial c_{p}}{\partial z}\right)-4\left(x a_{p}+y b_{p}+z c_{p}\right)\right\}\right. \\
& \left.+u^{p+2}\left(\frac{\partial a_{p}}{\partial x}+\frac{\partial b_{p}}{\partial y}+\frac{\partial c_{p}}{\partial z}\right)\right] .
\end{aligned}
$$

Put $g_{p}=f^{\prime}\left(\frac{\partial a_{p}}{\partial x}+\frac{\partial b_{p}}{\partial y}+\frac{\partial c_{p}}{\partial z}\right)-4\left(x a_{p}+y b_{p}+z c_{p}\right)$ and $h_{p}=\frac{\partial a_{p}}{\partial x}+\frac{\partial b_{p}}{\partial y}+\frac{\partial c_{p}}{\partial z}$ for non-negative integer $p$. Then every $\bar{g} \in \hat{B}$ has the following expression.

$$
\bar{g}=\left(g_{0}+u^{2} h_{0}\right)+u\left(g_{1}+u^{2} h_{1}\right)+\cdots+u^{p}\left(g_{p}+u^{2} h_{p}\right)+\cdots .
$$

First recall that $H_{N P}^{3}\left(\mathbb{R}^{3}, \eta^{\prime}\right) \cong \mathbb{R}$ by Proposition 6.4. Hence for any nonnegative integer $p$, it holds that

$$
\left\{g_{p} \mid a_{p}, b_{p}, c_{p} \in \mathcal{F}^{\prime}\right\}=\mathcal{F}_{0}^{\prime} .
$$

If we put $W_{p}=\left\{g_{p}+u^{2} h_{p} \mid a_{p}, b_{p}, c_{p} \in \mathcal{F}^{\prime}\right\}$, then $\bar{g}$ is contained in $W_{0}+u W_{1}+$ $\cdots+u^{p} W_{p}+\cdots$. Note that $h_{p}$ is not completely determined by $g_{p}$. To show this precisely, let us consider the following linear partial differential equation with three unknown functions $a, b, c \in \mathcal{F}^{\prime}$ :

$$
\text { (*) } \quad f^{\prime}\left(\frac{\partial a}{\partial x}+\frac{\partial b}{\partial y}+\frac{\partial c}{\partial z}\right)-4(x a+y b+z c)=0 .
$$

We define a subspace $\mathcal{F}_{0}^{\prime \prime}$ of $\mathcal{F}^{\prime}$ by

$$
\mathcal{F}_{0}^{\prime \prime}=\left\{\frac{\partial a}{\partial x}+\frac{\partial b}{\partial y}+\frac{\partial c}{\partial z} \mid \text { a triplet }(a, b, c) \text { is a solution of }(*)\right\} .
$$

Since $(a, b, c)$ is a solution of the differential equation $(*)$, there exist three functions $A, B, C \in \mathcal{F}^{\prime}$ such that

$$
\left\{\begin{array}{l}
a=f^{\prime}\left(C_{y}-B_{z}\right)+2(z B-y C), \\
b=f^{\prime}\left(A_{z}-C_{x}\right)+2(x C-z A), \\
c=f^{\prime}\left(B_{x}-A_{y}\right)+2(y A-x B) .
\end{array}\right.
$$


Recall that this fact is equivalent to $H_{f^{\prime}}^{2}=0$. Put $h=\frac{\partial a}{\partial x}+\frac{\partial b}{\partial y}+\frac{\partial c}{\partial z}$. If $h$ is an element of $\mathcal{F}_{0}^{\prime \prime}$, then it is clear that $h$ vanishes at the origin and hence $h \in \mathcal{F}_{0}^{\prime}$. Thus $\mathcal{F}_{0}^{\prime \prime}$ becomes a subspace of $\mathcal{F}_{0}^{\prime}$.

Let $g_{p}$ have the following two expressions:

$$
\begin{aligned}
g_{p} & =f^{\prime}\left(\frac{\partial a_{p}}{\partial x}+\frac{\partial b_{p}}{\partial y}+\frac{\partial c_{p}}{\partial z}\right)-4\left(x a_{p}+y b_{p}+z c_{p}\right) \\
& =f^{\prime}\left(\frac{\partial a_{p}^{\prime}}{\partial x}+\frac{\partial b_{p}^{\prime}}{\partial y}+\frac{\partial c_{p}^{\prime}}{\partial z}\right)-4\left(x a_{p}^{\prime}+y b_{p}^{\prime}+z c_{p}^{\prime}\right)
\end{aligned}
$$

for two triplets $\left(a_{p}, b_{p}, c_{p}\right)$ and $\left(a_{p}^{\prime}, b_{p}^{\prime}, c_{p}^{\prime}\right)$. Then we have

$$
\begin{aligned}
& f^{\prime}\left(\frac{\partial\left(a_{p}-a_{p}^{\prime}\right)}{\partial x}+\frac{\partial\left(b_{p}-b_{p}^{\prime}\right)}{\partial y}+\frac{\partial\left(c_{p}-c_{p}^{\prime}\right)}{\partial z}\right) \\
& \quad-4\left\{x\left(a_{p}-a_{p}^{\prime}\right)+y\left(b_{p}-b_{p}^{\prime}\right)+z\left(c_{p}-c_{p}^{\prime}\right)\right\}=0 .
\end{aligned}
$$

Hence

$$
h_{p}-h_{p}^{\prime}=\frac{\partial\left(a_{p}-a_{p}^{\prime}\right)}{\partial x}+\frac{\partial\left(b_{p}-b_{p}^{\prime}\right)}{\partial y}+\frac{\partial\left(c_{p}-c_{p}^{\prime}\right)}{\partial z}
$$

is an element of $\mathcal{F}_{0}^{\prime \prime}$, where $h_{p}^{\prime}=\frac{\partial a_{p}^{\prime}}{\partial x}+\frac{\partial b_{p}^{\prime}}{\partial y}+\frac{\partial c_{p}^{\prime}}{\partial z}$. Then it is easy to see that $h_{p}+\mathcal{F}_{0}^{\prime \prime}$, which denotes a coset of $h_{p}$ in $\mathcal{F}^{\prime} / \mathcal{F}_{0}^{\prime \prime}$, is uniquely determined by $g_{p}$. And each $W_{p}$ has the following expression:

$$
W_{p}=\left\{g_{p}+u^{2}\left(h_{p}+\mathcal{F}_{0}^{\prime \prime}\right) \mid g_{p} \in \mathcal{F}_{0}^{\prime}\right\} .
$$

Let $\phi_{p}: W_{p} \longrightarrow \mathcal{F}_{0}^{\prime}$ be a surjective linear mapping defined by $\phi_{p}\left(g_{p}+u^{2}\left(h_{p}+\right.\right.$ $\left.\left.\mathcal{F}_{0}^{\prime \prime}\right)\right)=g_{p}$. It is clear that $\phi_{p}$ is well-defined and that $g_{p}=0$ means $h_{p} \in \mathcal{F}_{0}^{\prime \prime}$. Hence $W_{p} / u^{2} \mathcal{F}_{0}^{\prime \prime} \cong \mathcal{F}_{0}^{\prime}$, and we have $W_{p} \cong \mathcal{F}_{0}^{\prime}+u^{2} \mathcal{F}_{0}^{\prime \prime}$. Now $\hat{B}$ becomes as follows. (Recall that $\mathcal{F}_{0}^{\prime \prime}$ is a subspace of $\mathcal{F}_{0}^{\prime}$.)

$$
\begin{aligned}
\hat{B}= & W_{0}+u W_{1}+u^{2} W_{2}+\cdots+u^{p} W_{p}+\cdots \\
\cong & \left(\mathcal{F}_{0}^{\prime}+u^{2} \mathcal{F}_{0}^{\prime \prime}\right)+u\left(\mathcal{F}_{0}^{\prime}+u^{2} \mathcal{F}_{0}^{\prime \prime}\right)+u^{2}\left(\mathcal{F}_{0}^{\prime}+u^{2} \mathcal{F}_{0}^{\prime \prime}\right) \\
& +\cdots+u^{p}\left(\mathcal{F}_{0}^{\prime}+u^{2} \mathcal{F}_{0}^{\prime \prime}\right)+\cdots \\
= & \mathcal{F}_{0}^{\prime}+u \mathcal{F}_{0}^{\prime}+u^{2} \mathcal{F}_{0}^{\prime}+\cdots+u^{p} \mathcal{F}_{0}^{\prime}+\cdots \\
= & \mathbb{R}[[u]] \mathcal{F}_{0}^{\prime} .
\end{aligned}
$$

Since

$$
\begin{aligned}
\hat{F} & =\mathcal{F}^{\prime}+u \mathcal{F}^{\prime}+u^{2} \mathcal{F}^{\prime}+\cdots \\
& =\left(\mathbb{R}+\mathcal{F}_{0}^{\prime}\right)+u\left(\mathbb{R}+\mathcal{F}_{0}^{\prime}\right)+u^{2}\left(\mathbb{R}+\mathcal{F}_{0}^{\prime}\right)+\cdots \\
& =\mathbb{R}[[u]] \oplus \mathbb{R}[[u]] \mathcal{F}_{0}^{\prime},
\end{aligned}
$$

we obtain that $\hat{F} / \hat{B} \cong \mathbb{R}[[u]]$. 
Let $T: \mathcal{F} \rightarrow \hat{F}$ be a linear mapping defined by $T(A)=\bar{A}$. For any $q \in T^{-1}(\hat{B})$, there exists $Q \in \hat{B}$ such that $T(q)=Q$. On the other hand, since $T(\mathcal{B})=\hat{B}$, there exists $q_{1} \in \mathcal{B}$ such that $T\left(q_{1}\right)=Q$. Hence $q-q_{1} \in \mathcal{I}$. By Lemma 6.7, we have $q \in \mathcal{B}$, and hence $T^{-1}(\hat{B})=\mathcal{B}$. Thus by Proposition 6.8 ,

$$
\mathcal{F} / \mathcal{B} \cong \hat{F} / \hat{B} \cong \mathbb{R}[[u]] .
$$

Now we summarize the results obtained in this section.

Theorem 6.9. Let $\eta=\left(x^{2}+y^{2}+z^{2}+u^{2}\right) \frac{\partial}{\partial x} \wedge \frac{\partial}{\partial y} \wedge \frac{\partial}{\partial z}$ be a NambuPoisson tensor on $\mathbb{R}^{4}(x, y, z, u)$. Then

$$
\begin{aligned}
& H_{N P}^{0}\left(\mathbb{R}^{4}, \eta\right) \cong C^{\infty}(\mathbb{R}), \\
& H_{N P}^{1}\left(\mathbb{R}^{4}, \eta\right) \cong \mathbb{R}[[u]], \\
& H_{N P}^{2}\left(\mathbb{R}^{4}, \eta\right)=0, \\
& H_{N P}^{3}\left(\mathbb{R}^{4}, \eta\right) \cong \mathbb{R}[[u]], \\
& H_{N P}^{k}\left(\mathbb{R}^{4}, \eta\right)=0, \quad k \geq 4 .
\end{aligned}
$$

\section{References}

[1] Abe, K. and Fukui, K., On the first homology of the group of diffeomorphisms of smooth orbifolds with isolated singularities, Preprint.

[2] de Rham, G., Sur la division de formes et de courants par une forme linéaire, Comment. Math. Helv., 28 (1954), 346-352.

[3] Dufour, J-P. and Zung, N. T., Linearization of Nambu structures, Compositio Math., 117 (1999), 77-98.

[4] Fukuda, T. and Janeczko, S., Smooth integrability of implicit differential systems, Max Planck Institut für Mathematik Preprint series, 78 (2002).

[5] Gautheron, P., Some remarks concerning Nambu mechanics, Lett. Math. Phys., 37 (1996), 103-116.

[6] Grabowski, J. and Marmo, G., On Filippov algebroids and multiplicative Nambu-Poisson structures, Differential Geom. Appl., 12 (2000), 35-50.

[7] Ibáñez, R., et al., Duality and modular class of a Nambu-Poisson structure, J. Phys. A, 34 (2001), 3623-3650.

[8] Mather, J., Solutions of generic linear equations, Proceedings of the conference on Dynamical systems, (ed. by M.M. Peixoto) Academic Press, (1973), 185-193.

[9] Monnier, P., Computations of Nambu-Poisson cohomologies, Int. J. Math. Math. Sci., 26 (2001), 65-81.

[10] Nakanishi, N., On Nambu-Poisson manifolds, Rev. Math. Phys., 10 (1998), 499-510.

[11] , Nambu-Poisson tensors on Lie groups, Banach Center Publ., 51 (2000), 243249.

[12] Narasimhan, R., Analysis on Real and Complex Manifolds, North-Holland Publishing Co. (1968).

[13] Roche, C. A., Cohomologie relative dans le domaine réel, Ph.D thesis, Université de Grenoble (1982).

[14] Takhtajan, L., On foundation of the generalized Nambu mechanics, Comm. Math. Phys., 160 (1994), 295-315. 